\title{
Synthesis of new 2-substituted 3-amino-4-hydroxymethylthiophenes through intramolecular nitrile oxide cycloaddition processes and $\mathrm{N}, \mathrm{O}$-bond cleavage
}

\author{
Venerando Pistarà, ${ }^{a *}$ Antonino Corsaro, ${ }^{a}$ Maria A. Chiacchio, ${ }^{\text {a Graziella Greco, }}{ }^{\text {a }}$ \\ and Paolo Quadrellib \\ ${ }^{a}$ Dipartimento di Scienze Chimiche, Università degli Studi di Catania \\ Viale A. Doria 6, I-95125 Catania, Italy \\ ${ }^{b}$ Dipartimento di Chimica Organica, Università degli Studi di Pavia \\ Via Taramelli 10, I-27100 Pavia, Italy \\ E-mail: vpistara@unict.it
}

\section{This paper is dedicated to Professor Heinz Heimgartner on the occasion of his 70th birthday}

\begin{abstract}
A synthesis of new 2-substituted 3-amino-4-hydroxymethylthiophenes 7 is reported as a useful alternative to the long known oximation of oxothiophenes $\mathbf{8}$, followed by treatment with gaseous hydrochloric acid in a polar solvent. The synthesis consists of an INOC process of unsaturated sulfide nitrile oxides, obtained from the condensation of corresponding nitroalkenes and allylmercaptan and then dehydration, which leads to tetrahydrothieno[3,4-c]isoxazoline 4. N,OBond cleavage of $\mathbf{4}$ upon hydride reduction affords to thiophenes 7 of biological interest.
\end{abstract}

Keywords: N,O-Cleavage, INOC processes, sugar heterocycles, 2-substitued 3-amino-4hydroxymethylthiophenes

\section{Introduction}

Intramolecular nitrile oxide-olefin cycloaddition processes (INOC) ${ }^{1}$ have been of considerable synthetic and mechanistic interest, especially, as far as it concerns the resulting isoxazoline ring which can serve as a precursor of different functionalities (hydroxyketone, aminoalcohol and other functional groups) stereoselectively produced by cleavage of the $\mathrm{N}, \mathrm{O}$-bond (Scheme 1). These INOC reactions are particularly useful in the field of the functionalization of heterocycles as they lead to isoxazoline-fused heterocycles, which, after selective scission of the isoxazoline 
nucleus, afford to heterocycles containing two functional groups which are otherwise difficult to introduce into the heterocycle, but, generally, easily manipulable. ${ }^{2}$

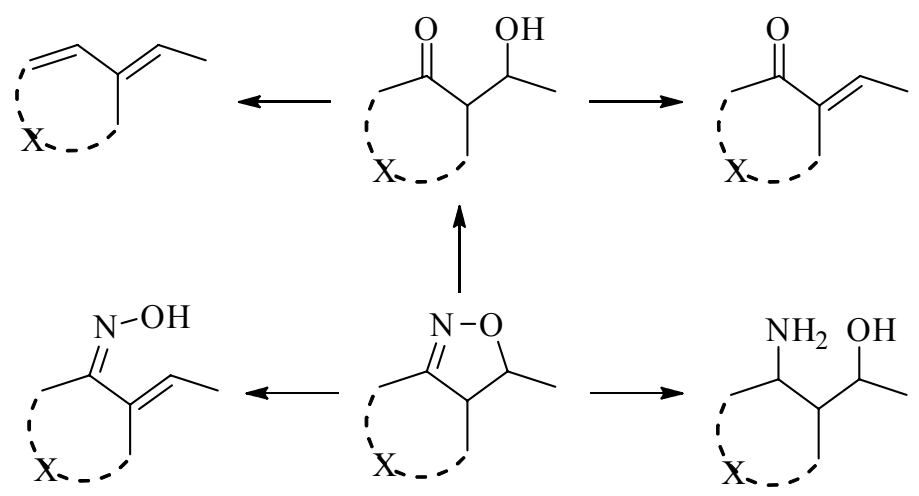

\section{Scheme 1}

In connection with our recent researches on thionucleoside syntheses ${ }^{3}$ we initially decided to apply this strategy of the INOC process for the preparation of 2-substituted 3-amino-4hydroxymethyltetrahydrothiophene nucleobases ${ }^{4}$ (Figure 1).

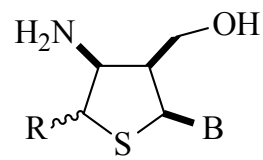

$$
\begin{aligned}
& \mathrm{B}=\text { thymine, cytosine, uracil, guanine, adenine } \\
& \mathrm{R}=\text { alkyl, phenyl, sugar groups }
\end{aligned}
$$

Figure 1. Structure of target nucleoside analogues.

Thus, as the first attempt, the treatment of a mixture of nitromethane, piperidine, and allyl mercaptan with acetaldehyde 1a at room temperature gave the unsaturated nitro sulfide $\mathbf{2 a}$ which affords the corresponding unisolable unsaturated nitrile oxide 3a by dehydration with phenyl isocyanate in the presence of triethylamine according to Mukaiyama ${ }^{5}$ (Scheme 2). This latter spontaneously undergoes a stereoselective INOC process to tetrahydrothieno[3,4-c]isoxazoline 4a.

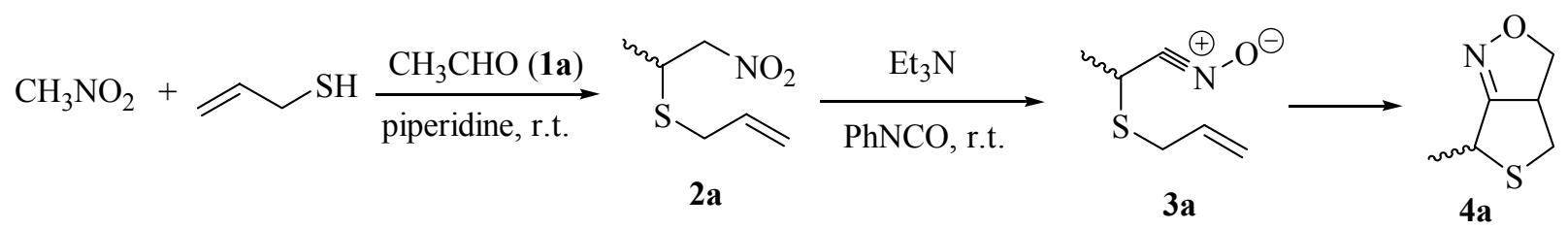

\section{Scheme 2}


Subsequently, with the aim of introducing into the free position of the bicyclic thioether the acetoxy group, to be substituted by pyrimidine or purine bases, $4 \mathbf{a}$ was oxidized to a corresponding mixture of diastereomeric sulfoxides $\mathbf{5} \mathbf{a}^{6}$ by periodic acid in the presence of ferric chloride, ${ }^{7}$ and subsequently subjected to the action of refluxing acetic anhydride ${ }^{8}$ (the Pummerer reaction conditions) (Scheme 3).

Against our expectations, instead of 4-acetoxy-6-methyltetrahydrothieno[3,4-c]isoxazoline, the oxidation of $\mathbf{4 a}$ followed by treatment with acetic anhydride afforded isoxazolinothiophene 6a in a moderate yield $(62 \%)$.

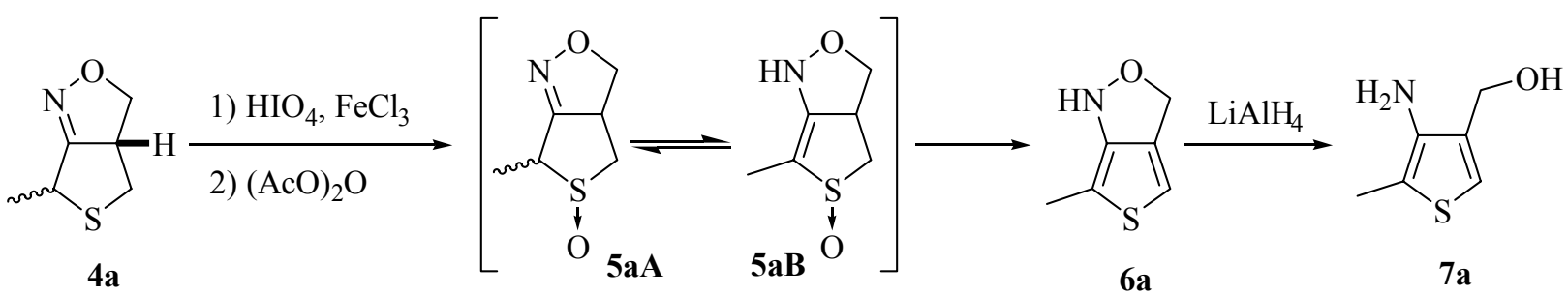

\section{Scheme 3}

Compounds 6a clearly derives from the formation of a mixture of diastereomeric thiophene oxides $\mathbf{5 a A}$, existing also as their tautomers $\mathbf{5 a B}$, which undergo a proton transposition from C-6 to $\mathrm{N}$, and aromatization of thiophene ring by loss of acetic acid.

Even so, isoxazolinothiophene 6a was converted by us into 3-amino-4-hydroxymethyl-2methylthiophene $\mathbf{7} \mathbf{a}^{9}$ upon reduction with lithium aluminium hydride by considering their potential biological properties, particularly when the amino group is substituted. ${ }^{10}$ In this paper we have extended the scope of the above reaction sequence to other derivatives $\mathbf{7 b} \mathbf{b} \mathbf{i}$ with several groups, such as ethyl, benzyl, and phenyl group, and also some sugar groups, linked to the 2 position of the ring, because 2-substituted-4-hydroxymethyl-3-aminotiophenes 7 are useful as intermediates in the synthesis of thiophene derivatives of pharmaceuticals and agrochemicals. ${ }^{10}$

These new derivatives 7 can also be obtained by reduction with lithium aluminium hydride in ether from the corresponding 3-amino-4-alkoxycarbonylthiophenes 9, which, in their turn, are for a long time known and are synthesized by means of a reaction sequence consisting of Salkylation of alkyl 3-mercapto propanoates in sodium methoxide-methanol with alkyl 2-bromoalkanoate at low temperature in moderate to good yield. These resulting thioethers are then cyclized with sodium methoxide at r.t. to give oxothiophenes 8 (Scheme 4), which are oximated and then treated with gaseous hydrochloric acid in a polar solvent, usually acetonitrile, to give to 2-substituted-3-amino-4-alkoxycarbonylthiophene hydrochlorides $\mathbf{9}^{11}$ 


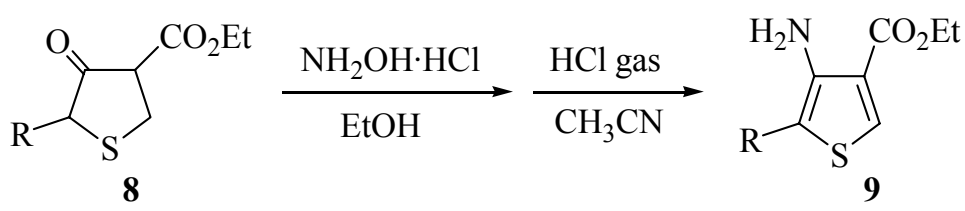

\section{Scheme 4}

In an alternate preparation, compounds 9 were obtained as hydrochloride salts in high yield from thiophenecarboxylates by mediated oxidation with sulfuryl chloride ${ }^{12}$ and furthermore they were also prepared by dehydrogenation of dihydrothiophenes in a two-step process involving treatment with hydrogen peroxide to give the dihydrothiophene $S$-oxide, and treatment of the oxides with acids. $^{13}$

\section{Results and Discussion}

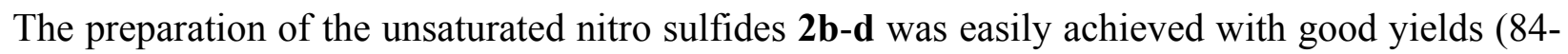
94\%) by following the Hassner ${ }^{4 a}$ work, which started from preformed nitroalkenes ${ }^{14}$ and allyl mercaptan in the presence of piperidine at room temperature. In a second step, nitro unsaturated sulfides 2b-d were converted on treatment with phenyl isocyanate-triethylamine ${ }^{5}$ into the unsaturated nitrile oxides $\mathbf{3 b}$-d, which, without to be isolated, underwent spontaneous cycloaddition to the fused diastereomeric isoxazolines $\mathbf{4 b - d}$ in excellent yields $(68-80 \%)$. In our hands, the diastereomeric compounds were not separated because our final target molecules were aromatic. Their structures, which were already known, ${ }^{4 a}$ with the exception of 4 d, were unambiguously assigned on the basis of their spectral data (see Experimental).
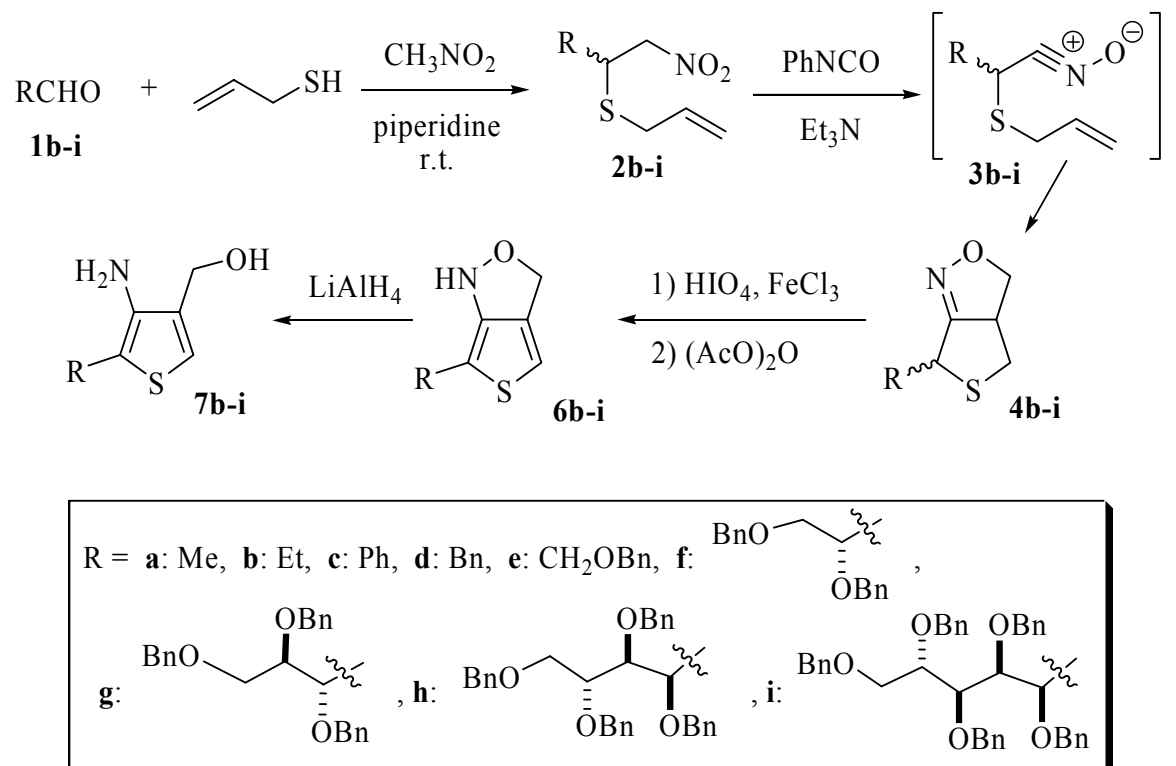

\section{Scheme 5}


Reaction of isoxazolinotetrahydrothiophenes $\mathbf{4 b}$-d with periodic acid in the presence of ferric chloride ${ }^{7}$ afforded to the corresponding unisolated tetrahydrothiophene oxides $\mathbf{5 b} \mathbf{b}-\mathbf{d}$, which subsequently gave bicyclic isoxazolinothiophenes 6b-d (70-75\%) upon treatment with acetic anhydride. The final reduction of 6a-d with lithium aluminium hydride led to expected new 2substituted 4-hydroxymethyl-3-aminotiophenes 7a-d, which were obtained in modest yields (55$62 \%$ ) due to their apparent instability for the presence of the free amino group. Therefore, they were not readily purified, but dissolved in absolute alcohol and added of gaseous hydrogen chloride. By addition of absolute ether to the reaction mixture, a initial crop (25-28\%) of the corresponding chlorides precipitated and after standing one more day, from the filtrate an additional $24-26 \%$ (total $49-54 \%$ ) of the salt deposited.

Some other attempts were conducted in the presence of weak oxidant reagents, which are reported in literature to afford only sulfoxide, without over-oxidation, such as sodium periodate supported on wet silica gel, ${ }^{15}$ hydrogen peroxide and $N$-hydroxysuccinimide, ${ }^{16}$ nitric acid in the presence of supported phosphoric anhydride on silica gel under solvent free conditions, ${ }^{17} o^{-}$ iodoxybenzoic acid and tetraethylammonium bromide as a catalyst ${ }^{18}$ but their further reactions in the presence of acetic anhydride led all to minor yields of 7a-d (50-56\%). By oxidation of $4 \mathbf{a}$ with 3-chloroperbenzoic acid, in his work, Hassner ${ }^{4 a}$ obtained unstable thiophene oxide which epimerized and partially decomposed on chromatography.

The new glycosides 7e-i were also prepared in moderate yield (54-60\%) within a research program directed to the synthesis of thiophenes derivatives with polyhydroxylated alkyl side chains in position 2. Having a number of useful properties, which include chirality, rigidity, lipophilicity, hydrophilicity, and flexibility in one system, polyhydroxyalkyl thiophenes are quite interesting scaffolds to utilize in the synthesis of a variety of biologically interesting molecules. Our program is devoted to substitute the oxygen atom with the sulphur atom in furans derivatives with polyhydroxylated alkyl side chains, the biological properties of which are well known. ${ }^{19}$

Their synthesis was achieved starting from sugar nitroalkenes ${ }^{20} \mathbf{2 e - i}$, obtained by the corresponding benzylated sugars and nitromethane and following the same above pathway for 7a-d through 4e-i (60-76\%) and 6e-i (68-74\%), with a diastereomeric ratios 1.2:8.8, 1.4:8.6, for $\mathbf{2 f}$ and $\mathbf{2 g}$, and 2.2:7.8 and 1.6:8.4 for $\mathbf{2 h}$ and $\mathbf{2 i}$, respectively.

In contrast with the 1:1 ratio of alkyl thiophenes $\mathbf{4 a - c}$, it is noteworthy that the cyclizations of phenyl and sugar derivatives 3d-i led to mixtures of trans-cis diastereomers $4 \mathbf{d}-\mathbf{i}$, ${ }^{1} \mathrm{H}$ NMR spectra of which showed the trans-diastereomer to be from 1.5 to 2.0 times the cis-diastereomer pointing out that steric factors play a determining role during the intramolecular cycloaddition.

The structures of the cyclised products $6 \mathbf{a}-\mathbf{i}$ were established by their IR and ${ }^{1} \mathrm{H}$ and ${ }^{13} \mathrm{C}$ NMR spectral data. For instance, in the case of compound $6 \mathbf{6}$, its ${ }^{1} \mathrm{H}$ NMR spectrum shows the characteristic signals of 4-thiophene proton at $6.31 \mathrm{ppm}$ and 3-methylene, benzyloxymethylene and benzylmethylene protons at 4.13,4.91, and $4.33 \mathrm{ppm}$, respectively. The corresponding signals of carbons in its ${ }^{13} \mathrm{C}$ NMR spectrum are found at $66.4,64.2$ and 73.7 , respectively, together with the presence of four aromatic carbons of which C-7a carbon is at $149.7 \mathrm{ppm}$. The 
IR spectroscopy was of great help for the identification of compounds 7, as they show a large broad absorption band included between 3400 and $3200 \mathrm{~cm}^{-1}$ for the amino and hydroxy groups, which appear as two broad signals in the regions 3.0-3.8 and 6.2-6.8 ppm in some of their ${ }^{1} \mathrm{H}$ NMR spectra. ${ }^{1} \mathrm{H}$ and ${ }^{13} \mathrm{C}$ NMR spectra of compounds 7e-i maintain the characteristic signals of sugar groups.

\section{Conclusions}

In conclusion, within our researches on thionucleoside syntheses, we have performed the preparation of new 2-substituted 3-amino-4-hydroxymethylthiophenes 7a-i, which appears to be a convenient and useful alternative to the long known oximation and conversion by gaseous hydrochloric acid of the corresponding 4-alkoxycarbonyl analogues. It provides moderate global yields, which, however, somewhat decrease by conversion into chlorides, but it starts from easily accessible materials.

\section{Experimental Section}

General. All melting points are uncorrected. Elemental analyses were done on a C. Erba 1106 elemental analyzer. IR spectra $\left(\mathrm{CHCl}_{3}\right.$ solutions) were recorded on an FT-IR Perkin-Elmer RX1. ${ }^{1} \mathrm{H}$ and ${ }^{13} \mathrm{C}$ NMR spectra were recorded on a Varian VNMR 200 in the specified deuterated solvents. Chemical shifts are expressed in parts per million from internal tetramethylsilane $(\delta)$. Merck silica gel $60(0.063$ and $0.200 \mathrm{~mm})$ was used for column chromatography and Merck precoated silica gel $60 \mathrm{~F} 254$ plates, $0.25 \mathrm{~mm}$ thickness, were used for analytical thin layer chromatography (TLC). Sugar compounds were detected with iodine vapours or $5 \%$ methanolic sulfuric acid spray followed by heating on a hot plate, while the other compounds were detected by UV $254 \mathrm{~nm}$.

Materials. $O$-Benzyl-D-sugar aldehydes 1e-i was prepared starting from the corresponding diethyl dithioacetals ${ }^{21}$ according to procedure described by López-Herrera and Sarabia-Garcia, ${ }^{22}$ while sugar nitroalkenes according to procedure of Sowden. ${ }^{20}$ Unless otherwise stated, the other materials were obtained from commercial suppliers and used without further purification. All the solvents and reagents, if it was necessary, were made anhydrous according to literature procedures. ${ }^{23}$ The organic solutions obtained by extractive workup were washed with saturated brine, dried over anhydrous sodium sulfate, and evaporated concentrated to dryness with a rotary evaporator under reduced pressure.

The identification of samples from different experiments was secured by superimposable IR spectra. Working with allylmercaptan, it required a good hood, but the purified $\beta$-nitrosulfides and their cyclized derivatives are practically odourless. 


\section{General procedure for the preparation of $\beta$-nitroalkyl sulfides (2a-i)}

A mixture of $1 \mathrm{mmol}$ of nitroalkene 1a-i, $1.1 \mathrm{mmol}$ of allyl mercaptan, and $0.1 \mathrm{~mol}$ of piperidine in $10 \mathrm{~mL}$ of THF was stirred for $1 \mathrm{~h}$ at $20^{\circ} \mathrm{C}$ according to literature procedure. ${ }^{24}$ Heat evolved and the mixture was refluxed for $2 \mathrm{~h}\left(80^{\circ} \mathrm{C}\right.$, oil bath). THF was added and the resulting solution was washed with dilute hydrochloric acid, water, and brine and finally it was dried $\left(\mathrm{MgSO}_{4}\right)$. After evaporation of the solvent under reduced pressure, the oily residue was chromatographed on silica gel (eluant: cyclohexane:ether $=10: 1$ ) to give a diastereomeric mixture of $\beta$-nitroalkyl sulfides $\mathbf{2 f}, \mathbf{2 g}, \mathbf{2} \mathbf{h}$ and $\mathbf{2 i}$, in the ratios 1.2:8.8, 1.4:8.6, 2.2:7.8 and 1.6:8.4, respectively, as it appeared from the integrations of the $\mathrm{CH}$-S proton signals of the ${ }^{1} \mathrm{H}$ NMR spectra performed on the corresponding crude reaction mixtures. The ${ }^{1} \mathrm{H}$ NMR data, described below, report chemical shifts of major diastereomers, but their absolute stereochemistry was not determined because the final target molecules were aromatic.

2-(Allylthio)-1-nitro-propane 2a, -butane 2b, -2-phenylethane 2c are known compounds and were prepared in 92,90 and $94 \%$ yield, respectively, from the corresponding nitroalkenes. ${ }^{4 a}$

$(\boldsymbol{R}, \boldsymbol{S})$-2-(Allylthio)-1-nitro-3-phenylpropane (2d). Colourless oil, yield 90\%, IR $\left(v_{\max } \mathrm{cm}^{-1}\right)$ : 2250, $1490\left(\mathrm{NO}_{2}\right) .{ }^{1} \mathrm{H}$ NMR $\left(500 \mathrm{MHz}, \mathrm{CD}_{3} \mathrm{CN}\right), \delta_{\mathrm{H}} 2.85$ (dd, $1 \mathrm{H}, J=12.0,5.0 \mathrm{~Hz}, \mathrm{CH}_{2} \mathrm{Ph}$ ), $2.94\left(\mathrm{dd}, 1 \mathrm{H}, J=12.0,6.5 \mathrm{~Hz}, \mathrm{CH}_{2} \mathrm{Ph}\right), 3.28\left(1 \mathrm{H}, \mathrm{dd}, J=12.5,7.3 \mathrm{~Hz}, \mathrm{CH}_{2} \mathrm{~S}\right), 3.31(1 \mathrm{H}, \mathrm{dd}, J=$ 12.5, $7.3 \mathrm{~Hz}, \mathrm{CH}_{2} \mathrm{~S}$ ), 3.54 (1H, m, CHS), 4.43 (dd, $1 \mathrm{H}, J=12.5,7.0 \mathrm{~Hz}, \mathrm{CH}_{2} \mathrm{NO}_{2}$ ), 4.65 (dd, $1 \mathrm{H}$, $\left.J=12.5,2.0 \mathrm{~Hz}, \mathrm{CH}_{2} \mathrm{NO}_{2}\right), 5.19\left(1 \mathrm{H}, \mathrm{dd}, J=10.0,1.5 \mathrm{~Hz},=\mathrm{CH}_{2}\right), 5.21(\mathrm{dd}, 1 \mathrm{H}, J=17.0,1.5$ $\left.\mathrm{Hz},=\mathrm{CH}_{2}\right), 5.75(1 \mathrm{H}, \mathrm{ddt}, J=17.0,10.0,7.3 \mathrm{~Hz},=\mathrm{CH}), 7.20-7.28(5 \mathrm{H}, \mathrm{m}$, phenyl $\mathrm{H}) .{ }^{13} \mathrm{C} \mathrm{NMR}$ $\left(50 \mathrm{MHz}, \mathrm{CDCl}_{3}\right), \delta_{\mathrm{C}} 36.2,40.1,42.9,79.5,118.4,125.5,128.3,129.5,132.1,136.4$. Anal. Calcd. for $\mathrm{C}_{12} \mathrm{H}_{15} \mathrm{NO}_{2} \mathrm{~S}$ (237.31): C, 60.73; H, 6.37; N, 5.90, Found: C, 60.77; H, 6.31; N, 5.08.

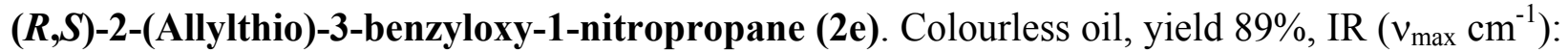
2240, $1480\left(\mathrm{NO}_{2}\right) .{ }^{1} \mathrm{H}$ NMR $\left(500 \mathrm{MHz}, \mathrm{CD}_{3} \mathrm{CN}\right), \delta_{\mathrm{H}} 3.16\left(1 \mathrm{H}, \mathrm{dd}, J=12.0,7.5 \mathrm{~Hz}, \mathrm{CH}_{2} \mathrm{~S}\right), 3.18$ $\left(1 \mathrm{H}, \mathrm{dd}, J=12.0,7.5 \mathrm{~Hz}, \mathrm{CH}_{2} \mathrm{~S}\right), 3.42$ (m, 1H, CHS), $3.50\left(\mathrm{dd}, 1 \mathrm{H}, J=11.5,5.0 \mathrm{~Hz}, \mathrm{CH}_{2} \mathrm{O}\right.$ ), $3.58\left(\mathrm{dd}, 1 \mathrm{H}, J=11.5,6.0 \mathrm{~Hz}, \mathrm{CH}_{2} \mathrm{O}\right), 4.51\left(\mathrm{~s}, 2 \mathrm{H}, \mathrm{CH}_{2} \mathrm{Ph}\right), 4.38(\mathrm{dd}, 1 \mathrm{H}, J=12.0,6.0 \mathrm{~Hz}$, $\left.\mathrm{CH}_{2} \mathrm{NO}_{2}\right), 4.45\left(\mathrm{dd}, 1 \mathrm{H}, J=12.0,9.0 \mathrm{~Hz}, \mathrm{CH}_{2} \mathrm{NO}_{2}\right), 5.17\left(\mathrm{dd}, 1 \mathrm{H}, J=10.0,1.6 \mathrm{~Hz},=\mathrm{CH}_{2}\right), 5.22$ $\left(1 \mathrm{H}, \mathrm{dd}, J=16.5,1.6 \mathrm{~Hz},=\mathrm{CH}_{2}\right), 5.69(1 \mathrm{H}, \mathrm{ddt}, J=16.5,10.0,7.5 \mathrm{~Hz},=\mathrm{CH}), 7.24-7.37(\mathrm{~m}, 5 \mathrm{H}$, phenyl H). ${ }^{13} \mathrm{C}$ NMR $\left(50 \mathrm{MHz}, \mathrm{CDCl}_{3}\right), \delta_{\mathrm{C}} 35.8,42.1,69.8,77.4,77.5,116.4,125.5,127.3$, 128.5, 132.1, 137.4. Anal. Calcd. for $\mathrm{C}_{13} \mathrm{H}_{17} \mathrm{NO}_{3} \mathrm{~S}$ (267.34): C, 58.40; H, 6.41; N, 5.24, Found: C, 58.47; H, 6.39; N, 5.31 .

Rac-2-(3R)-2-(Allylthio)-3,4-bis(benzyloxy)-1-nitrobutane (2f). Colourless oil, yield 87\%, IR $\left(v_{\max } \mathrm{cm}^{-1}\right): 2260,1490\left(\mathrm{NO}_{2}\right) .{ }^{1} \mathrm{H}$ NMR $\left(500 \mathrm{MHz}, \mathrm{CD}_{3} \mathrm{CN}\right), \delta_{\mathrm{H}} 3.21(1 \mathrm{H}, \mathrm{dd}, J=12.5,7.5 \mathrm{~Hz}$, $\mathrm{CH}_{2} \mathrm{~S}$ ), $3.23\left(1 \mathrm{H}, \mathrm{dd}, J=12.5,7.5 \mathrm{~Hz}, \mathrm{CH}_{2} \mathrm{~S}\right), 3.31$ (m, 1H, CHS), 3.43 (dd, $1 \mathrm{H}, J=10.5,7.5$ $\mathrm{Hz}, \mathrm{CH}_{2} \mathrm{O}$ ), 3.55 (dd, 1H, $J=10.5,1.5 \mathrm{~Hz}, \mathrm{CH}_{2} \mathrm{O}$ ), 3.72 (ddd, $1 \mathrm{H}, J=7.5,5.5,1.5 \mathrm{~Hz}, \mathrm{CHO}$ ), $4.33\left(\mathrm{dd}, 1 \mathrm{H}, J=12.0,6.5 \mathrm{~Hz}, \mathrm{CH}_{2} \mathrm{NO}_{2}\right), 4.51\left(\mathrm{dd}, 1 \mathrm{H}, J=12.0,9.5 \mathrm{~Hz}, \mathrm{CH}_{2} \mathrm{NO}_{2}\right), 4.55-4.68$ (AB system, $2 \mathrm{H}, J=11.5 \mathrm{~Hz}, \mathrm{OCH}_{2} \mathrm{Ph}$ ), 4.64-4.75 (AB system, $2 \mathrm{H}, J=11.5 \mathrm{~Hz}, \mathrm{OCH}_{2} \mathrm{Ph}$ ), 5.16 $\left(\mathrm{dd}, 1 \mathrm{H}, J=10.5,1.5 \mathrm{~Hz},=\mathrm{CH}_{2}\right), 5.19\left(\mathrm{dd}, 1 \mathrm{H}, J=16.5,1.5 \mathrm{~Hz},=\mathrm{CH}_{2}\right), 5.82(\mathrm{ddt}, 1 \mathrm{H}, J=16.5$, $10.5,7.5 \mathrm{~Hz},=\mathrm{CH}), 7.22-7.38\left(\mathrm{~m}, 10 \mathrm{H}\right.$, phenyl H). ${ }^{13} \mathrm{C} \mathrm{NMR}\left(50 \mathrm{MHz}, \mathrm{CDCl}_{3}\right), \delta_{\mathrm{C}} 36.3,43.4$, 
$69.8,73.1,73.2,73.5,76.2,77.8,81.9,118.4,126.7,127.5,127.8,127.9,128.1,128.2,128.3$, 128.6, 128.8, 132.5, 136.7, 137.9. Anal. Calcd. for $\mathrm{C}_{21} \mathrm{H}_{25} \mathrm{NO}_{4} \mathrm{~S}$ (387.49): C, 65.09; $\mathrm{H}, 6.50$; $\mathrm{N}$, 3.61, Found: C, 65.21; H, 6.59; N, 3.53.

Rac-2-(3R,4R)-2-(Allylthio)-3,4,5-tris(benzyloxy)-1-nitropentane (2g). Colourless oil, yield $88 \%$, IR $\left(v_{\max } \mathrm{cm}^{-1}\right): 2240,1470\left(\mathrm{NO}_{2}\right) .{ }^{1} \mathrm{H}$ NMR $\left(500 \mathrm{MHz}, \mathrm{CD}_{3} \mathrm{CN}\right), \delta_{\mathrm{H}} 3.34(\mathrm{dd}, 1 \mathrm{H}, J=12.5$, $7.5 \mathrm{~Hz}, \mathrm{CH}_{2} \mathrm{~S}$ ), 3.40 (dd, $1 \mathrm{H}, J=12.5,7.5, \mathrm{~Hz}, \mathrm{CH}_{2} \mathrm{~S}$ ), 3.41 (m, 1H, CHS), 3.60 (dd, $1 \mathrm{H}, J=6.5$, 6.0, Hz, SCHCH), 3.69 (dd, 1H, $\left.J=10.5,6.5 \mathrm{~Hz}, \mathrm{OCHCH}_{2}\right), 3.73(\mathrm{dd}, 1 \mathrm{H}, J=10.5,2.5 \mathrm{~Hz}$, $\mathrm{OCHCH}_{2}$ ), $4.11\left(\mathrm{ddd}, 1 \mathrm{H}, J=7.0,6.5,2.5 \mathrm{~Hz}, \mathrm{OCHCH}_{2}\right), 4.36(\mathrm{dd}, 1 \mathrm{H}, J=13.0,5.5, \mathrm{~Hz}$, $\mathrm{CH}_{2} \mathrm{NO}_{2}$ ), 4.51-4.54 (AB system, $\left.2 \mathrm{H}, J=11.5 \mathrm{~Hz}, \mathrm{OCH}_{2} \mathrm{Ph}\right), 4.59$ (dd, $1 \mathrm{H}, J=10.0,5.5, \mathrm{~Hz}$, $\mathrm{CH}_{2} \mathrm{NO}_{2}$ ), 4.61-4.77 (AB system, $2 \mathrm{H}, J=11.5 \mathrm{~Hz}, \mathrm{OCH}_{2} \mathrm{Ph}$ ), 4.68-5.74 (AB system, $2 \mathrm{H}, J=$ $\left.12.0 \mathrm{~Hz}, \mathrm{OCH}_{2} \mathrm{Ph}\right), 5.17\left(\mathrm{dd}, 1 \mathrm{H}, J=15.5,1.5 \mathrm{~Hz},=\mathrm{CH}_{2}\right), 5.21\left(\mathrm{dd}, 1 \mathrm{H}, J=9.5,1.5 \mathrm{~Hz},=\mathrm{CH}_{2}\right)$, 5.87 (ddt, $1 \mathrm{H}, J=15.5,9.5,7.5 \mathrm{~Hz},=\mathrm{CH}), 7.23-7.37\left(\mathrm{~m}, 15 \mathrm{H}\right.$, phenyl H). ${ }^{13} \mathrm{C} \mathrm{NMR}(50 \mathrm{MHz}$, $\left.\mathrm{CDCl}_{3}\right), \delta_{\mathrm{C}} 36.2,44.0,72.7,73.4,73.9,76.7,78.4,118.4,127.1,127.3,127.8,128.0,128.3$, 128.7, 132.7, 136.6, 138.9, 139.1. Anal. Calcd. for $\mathrm{C}_{29} \mathrm{H}_{33} \mathrm{NO}_{5} \mathrm{~S}(507.64)$ : C, 68.61; H, 6.55; N, 2.76, Found: C, 68.67; H, 6.58; N, 2.73.

Rac-2-(3S,4S,5S)-2-Allyl-3,4,5,6-tetrakis(benzyloxy)-1-nitrohexane (2h). Colourless oil, yield 84\%, IR ( $\left.v_{\max } \mathrm{cm}^{-1}\right): 2250,1490\left(\mathrm{NO}_{2}\right) .{ }^{1} \mathrm{H} \mathrm{NMR}\left(500 \mathrm{MHz}, \mathrm{CD}_{3} \mathrm{CN}\right), \delta_{\mathrm{H}} 3.31(\mathrm{dd}, 1 \mathrm{H}, J=12.0$, $7.0 \mathrm{~Hz}, \mathrm{CH}_{2} \mathrm{~S}$ ), 3.39 (m, 1H, CHS), $3.43\left(\mathrm{dd}, 1 \mathrm{H}, J=12.0,7.0 \mathrm{~Hz}, \mathrm{CH}_{2} \mathrm{~S}\right), 3.61$ (dd, $1 \mathrm{H}, J=$ $\left.10.5,4.5 \mathrm{~Hz}, \mathrm{OCHCH}_{2}\right), 3.72\left(\mathrm{dd}, 1 \mathrm{H}, J=10.5,4.5 \mathrm{~Hz}, \mathrm{OCHCH}_{2}\right), 3.79(\mathrm{dd}, 1 \mathrm{H}, J=6.5,4.5$ $\mathrm{SCHCH}), 3.88\left(\mathrm{dd}, 1 \mathrm{H}, J=6.0,4.5 \mathrm{~Hz} \mathrm{OCHCH}_{2}\right), 4.28(\mathrm{dd}, 1 \mathrm{H}, J=6.0,4.5 \mathrm{~Hz}, \mathrm{OCHCH}), 4.37$ (dd, $1 \mathrm{H}, J=12.5,5.5 \mathrm{~Hz}, \mathrm{CH}_{2} \mathrm{NO}_{2}$ ), 4.41-4.62 (AB system, $2 \mathrm{H}, J=11.5 \mathrm{~Hz}, \mathrm{OCH}_{2} \mathrm{Ph}$ ), 4.48 (dd, $1 \mathrm{H}, J=10.0,5.5 \mathrm{~Hz}, \mathrm{CH}_{2} \mathrm{NO}_{2}$ ), 4.56-4.58 (AB system, $2 \mathrm{H}, J=11.5 \mathrm{~Hz}, \mathrm{OCH}_{2} \mathrm{Ph}$ ), 4.59-4.71 (AB system, $2 \mathrm{H}, J=11.5 \mathrm{~Hz}, \mathrm{OCH}_{2} \mathrm{Ph}$ ), 4.61-4.69 (AB system, $2 \mathrm{H}, J=11.5 \mathrm{~Hz}, \mathrm{OCH}_{2} \mathrm{Ph}$ ), 5.15 $\left(\mathrm{dd}, 1 \mathrm{H}, J=14.5,1.5 \mathrm{~Hz},=\mathrm{CH}_{2}\right), 5.23\left(\mathrm{dd}, 1 \mathrm{H}, J=9.5,1.5 \mathrm{~Hz},=\mathrm{CH}_{2}\right), 5.81(\mathrm{ddt}, 1 \mathrm{H}, J=14.5$, 9.5, 7.0 Hz, $=\mathrm{CH}), 7.21-7.36\left(\mathrm{~m}, 20 \mathrm{H}\right.$, phenyl H). ${ }^{13} \mathrm{C} \mathrm{NMR}\left(50 \mathrm{MHz}, \mathrm{CDCl}_{3}\right), \delta_{\mathrm{C}} 36.2,44.0$, 69.7, 72.0, 73.3, 73.4, 74.3, 77.6, 78.6, 118.4, 127.1, 127.3, 127.7, 128.0, 128.1, 128.2, 128.3, 128.4, 136.6, 137.3, 137.7, 138.2. Anal. Calcd. for $\mathrm{C}_{37} \mathrm{H}_{41} \mathrm{NO}_{6} \mathrm{~S}(627.79): \mathrm{C}, 70.79 ; \mathrm{H}, 5.68 ; \mathrm{N}$, 2.23, Found: C, 70.67; H, 5.61; N, 2.33.

Rac-2-(3R,4S,5R,6R)-2-Allyl-3,4,5,6,7-pentakis(benzyloxy)-1-nitroheptane (2i). Colourless oil, yield 84\%, IR ( $\left.v_{\max } \mathrm{cm}^{-1}\right): 2280,1460\left(\mathrm{NO}_{2}\right) .{ }^{1} \mathrm{H} \mathrm{NMR}\left(500 \mathrm{MHz}, \mathrm{CD}_{3} \mathrm{CN}\right), \delta_{\mathrm{H}} 3.36(\mathrm{dd}, 1 \mathrm{H}$, $J=12.5,7.0, \mathrm{~Hz}, \mathrm{CH}_{2} \mathrm{~S}$ ), 3.41 (m, 1H, CHS), 3.46 (dd, $1 \mathrm{H}, J=12.5,7.0 \mathrm{~Hz}, \mathrm{CH}_{2} \mathrm{~S}$ ), 3.55 (dd, $\left.1 \mathrm{H}, J=10.5,4.5 \mathrm{~Hz}, \mathrm{OCHCH}_{2}\right), 3.68\left(\mathrm{dd}, 1 \mathrm{H}, J=10.5,4.5 \mathrm{~Hz}, \mathrm{OCHCH}_{2}\right), 3.71(\mathrm{dd}, 1 \mathrm{H}, J=$ 4.5, $6.5 \mathrm{~Hz}, \mathrm{SCHCH}), 3.80\left(\mathrm{dd}, 1 \mathrm{H}, J=6.0,4.5 \mathrm{~Hz}, \mathrm{OCHCH}_{2}\right), 4.04(\mathrm{dd}, 1 \mathrm{H}, J=6.0,4.5 \mathrm{~Hz}$, $\mathrm{CHCHCH}), 4.11(\mathrm{dd}, 1 \mathrm{H}, J=6.0,4.5 \mathrm{~Hz}, \mathrm{OCHCH}), 4.36\left(\mathrm{dd}, 1 \mathrm{H}, J=12.5,5.5 \mathrm{~Hz}, \mathrm{CH}_{2} \mathrm{NO}_{2}\right.$ ), 4.38-4.52 (AB system, 2H, $J=11.5 \mathrm{~Hz}, \mathrm{OCH}_{2} \mathrm{Ph}$ ), 4.41 (dd, $1 \mathrm{H}, J=10.0,5.5 \mathrm{~Hz}, \mathrm{CH}_{2} \mathrm{NO}_{2}$ ), 4.46-4.78 (AB system, $2 \mathrm{H}, J=11.5 \mathrm{~Hz}, \mathrm{OCH}_{2} \mathrm{Ph}$ ), 4.51-4.68 (AB system, $2 \mathrm{H}, J=11.5 \mathrm{~Hz}$, $\mathrm{OCH}_{2} \mathrm{Ph}$ ), 4.57-4.63 (AB system, $2 \mathrm{H}, J=11.5 \mathrm{~Hz}, \mathrm{OCH}_{2} \mathrm{Ph}$ ), 4.59-4.69 (AB system, $2 \mathrm{H}, J=$ $\left.11.5 \mathrm{~Hz}, \mathrm{OCH}_{2} \mathrm{Ph}\right), 5.11\left(\mathrm{dd}, 1 \mathrm{H}, J=15.0,1.5 \mathrm{~Hz},=\mathrm{CH}_{2}\right), 5.21\left(\mathrm{dd}, 1 \mathrm{H}, J=9.0,1.5 \mathrm{~Hz},=\mathrm{CH}_{2}\right)$, 5.83 (ddt, $1 \mathrm{H}, J=15.0,9.0,7.0 \mathrm{~Hz},=\mathrm{CH}), 7.23-7.37\left(\mathrm{~m}, 25 \mathrm{H}\right.$, phenyl H). ${ }^{13} \mathrm{C} \mathrm{NMR}(50 \mathrm{MHz}$, $\left.\mathrm{CDCl}_{3}\right), \delta_{\mathrm{C}} 36.2,44.0,69.7,71.6,72.0,73.0,73.6,74.2,77.6,78.1,80.1,80.6,118.4,127.1$, 
$127.4,127.5,127.7,128.6,127.9,128.0,128.1,128,2,128.5,136.9,137.5,137.7,138.1,138.4$. Anal. Calcd. for $\mathrm{C}_{45} \mathrm{H}_{49} \mathrm{NO}_{7} \mathrm{~S}$ (747.93): C, 72.26; H, 6.60; N, 1.87, Found: C, 72.34; H, 6.65; N, 1.83.

General procedure for the generation of nitrile oxides (3a-i) from $\beta$-nitroalkyl sulfides and their intramolecular cycloadditions to 3,3a,4,6-tetrahydrothieno[3,4-c] isoxazoles (4a-i)

To a solution of $\beta$-nitroalkyl sulfides $(2 \mathrm{mmol})$ in $10 \mathrm{ml}$ of dry toluene, containing a few drops of trietylamine, phenyl isocyanate $(6 \mathrm{mmol})$ was added. The resulting solution was allowed to stand at room temperature for 3 days. Diphenylurea was filtered and the solvent was removed under reduced pressure. The obtained residue was chromatographed over silica gel (eluent: petroleum ether:ether $=2: 1$ ) to yield a nearly 1:1 mixture of diastereomers which were not separated because the target molecules were aromatic.

6-Methyl- (4a), 6-ethyl- (4b), and 6-phenyl-3,4-dihydro-3H,6H-thieno[3,4-c]isoxazole (4c) are known products ${ }^{4 \mathrm{a}}$ and were prepared in 80,74 , and $68 \%$ yield, respectively, from the corresponding $\beta$-nitroalkyl sulfides (2a-c).

Rac-6-benzyl-3a,4-dihydro-3H,6H-thieno[3,4-c]isoxazole (4d). Colourless oil, yield $72 \%$. ${ }^{1} \mathrm{H}$ NMR $\left(200 \mathrm{MHz}, \mathrm{CDCl}_{3}\right), \delta_{\mathrm{H}} 2.87\left(\mathrm{ddd}, 1 \mathrm{H}, J=5.0,9.0,12.0 \mathrm{~Hz}, \mathrm{CH}_{2} \mathrm{Ph}\right), 3.52(\mathrm{~m}, 2 \mathrm{H}$, $\mathrm{CH}_{2} \mathrm{CHCH}_{2}$ ), $3.64\left(\mathrm{~m}, 2 \mathrm{H}, \mathrm{SCH}_{2}\right), 4.23\left(\mathrm{dd}, 1 \mathrm{H}, J=5.0,9.0 \mathrm{~Hz}, \mathrm{CH}_{2} \mathrm{CHS}\right), 4.29-4.36(\mathrm{AB}$ system, $\left.2 \mathrm{H}, J_{\mathrm{AB}}=11.5 \mathrm{~Hz}, \mathrm{OCH}_{2}\right), 7.18-7.27\left(5 \mathrm{H}, \mathrm{m}\right.$, phenyl H). ${ }^{13} \mathrm{C} \mathrm{NMR}\left(50 \mathrm{MHz}, \mathrm{CDCl}_{3}\right), \delta_{\mathrm{C}}$ 32.4, 36.5, 49.0, 58.9, 74.6, 125.8, 128.1, 129.5, 137.3, 157.7. Anal. Calcd. for $\mathrm{C}_{12} \mathrm{H}_{13} \mathrm{NOS}$ (219.30): C, 65.72; H, 5.97; N, 6.39, Found: C, 65.77; H, 5.91; N, 6.38.

Rac-6-[(benzyloxy)methyl]-3a,4-dihydro-3H,6H-thieno[3,4-c]isoxazole (4e). Colourless oil, yield 74\%. ${ }^{1} \mathrm{H}$ NMR $\left(200 \mathrm{MHz}, \mathrm{CDCl}_{3}\right), \delta_{\mathrm{H}} 3.43\left(\mathrm{~m}, 3 \mathrm{H}, \mathrm{SCH}_{2} \mathrm{CH}\right), 3.58(\mathrm{dd}, 2 \mathrm{H}, J=2.5,7.0$ $\left.\mathrm{Hz}, \mathrm{SCHCH}_{2}\right), 4.34\left(\mathrm{~m}, 2 \mathrm{H}, \mathrm{NOCH}_{2}\right), 4.69\left(\mathrm{~m}, 2 \mathrm{H}, \mathrm{CH}_{2} \mathrm{Ph}\right), 4.70$ (dd, $1 \mathrm{H}, J=2.5,7.0 \mathrm{~Hz}$, $\mathrm{CH}_{2} \mathrm{CHS}$ ), 7.22-7.32 (m, 5H, phenyl H). ${ }^{13} \mathrm{C} \mathrm{NMR}\left(50 \mathrm{MHz}, \mathrm{CDCl}_{3}\right), \delta_{\mathrm{C}} 32.4,36.5,49.0,58.9$, 74.6, 125.8, 128.1, 129.5, 137.3, 157.7. Anal. Calcd. for $\mathrm{C}_{13} \mathrm{H}_{15} \mathrm{NOS}$ (249.32): C, 62.62; H, 6.06; N, 5.63, Found: C, 62.67; H, 6.01; N, 5.68.

Rac-6-[(1R)-1,2-bis(benzyloxy)ethyl]-3a,4-dihydro-3H,6H-thieno[3,4-c]isoxazole (4f). Colourless oil, yield 70\%. ${ }^{1} \mathrm{H}$ NMR $\left(200 \mathrm{MHz}, \mathrm{CDCl}_{3}\right.$ ), $\delta_{\mathrm{H}}$ 3.44-3.60 (AB system, $2 \mathrm{H}, J=11.5$ $\left.\mathrm{Hz} \mathrm{CH}_{2} \mathrm{CHCH}\right), 3.49$ (m, 3H, SCH $\mathrm{CH}_{2}, 3.65$ (m, 1H, $\left.\mathrm{CH}_{2} \mathrm{CHCH}\right), 4.40\left(\mathrm{~m}, 2 \mathrm{H}, \mathrm{NOCH}_{2}\right), 4.50$ $\left(\mathrm{s}, 2 \mathrm{H}, \mathrm{CH}_{2} \mathrm{OCH} \mathrm{P}_{2} \mathrm{Ph}\right), 4.81\left(\mathrm{~d}, 1 \mathrm{H}, J=5.5 \mathrm{~Hz}, \mathrm{CHS}\right.$ ), 5.02-5.12 (AB system, $2 \mathrm{H}, J_{\mathrm{AB}}=11.5 \mathrm{~Hz}$, $\left.\mathrm{PhCH} \mathrm{H}_{2} \mathrm{OCH}\right), 7.18-7.34\left(\mathrm{~m}, 10 \mathrm{H}\right.$, phenyl H). ${ }^{13} \mathrm{C} \mathrm{NMR}\left(50 \mathrm{MHz}, \mathrm{CDCl}_{3}\right), \delta_{\mathrm{C}} 28.3,50.0,58.8$, 39.1, 72.9, 73.0, 79.6, 127.2, 127.5, 127.8, 128.1, 128.5, 128.8, 137.7, 137.4, 156.0. Anal. Calcd. for $\mathrm{C}_{21} \mathrm{H}_{23} \mathrm{NO}_{3} \mathrm{~S}$ (369.47): C, 68.27; H, 6.27; N, 3.79, Found: C, 68.31; H, 6.21; N, 3.71.

Rac-6-[(1R,2R)-1,2,3-tris(benzyloxy)propyl]-3a,4-dihydro-3H,6H-thieno[3,4-c]isoxazole (4g). Colourless oil, yield 70\%. ${ }^{1} \mathrm{H}$ NMR (200 MHz, $\left.\mathrm{CDCl}_{3}\right), \delta_{\mathrm{H}} 3.45\left(\mathrm{~m}, 3 \mathrm{H}, \mathrm{SCH}_{2} \mathrm{CH}\right), 3.55$ $(\mathrm{m}, 1 \mathrm{H}, \mathrm{SCHCH}), 3.84\left(\mathrm{~m}, 2 \mathrm{H}, \mathrm{BnOCH}_{2} \mathrm{CH}\right), 4.10\left(\mathrm{~m}, 1 \mathrm{H}, \mathrm{CH}_{2} \mathrm{CHCH}\right), 4.30\left(\mathrm{~m}, 1 \mathrm{H}, \mathrm{NOCH}_{2}\right)$, $4.37\left(\mathrm{~m}, 1 \mathrm{H}, \mathrm{NOCH}_{2}\right), 4.70\left(\mathrm{~s}, 2 \mathrm{H}, \mathrm{CH}_{2} \mathrm{OCH}{ }_{2} \mathrm{Ph}\right), 4.75$ (d, 1H, J=5.5 Hz, CHS), 4.86-4.96 (AB system, $2 \mathrm{H}, J=11.5 \mathrm{~Hz}, \mathrm{PhCH}_{2} \mathrm{OCH}$ ), 5.05-5.15 (AB system, $2 \mathrm{H}, J=11.5 \mathrm{~Hz}, \mathrm{PhCH}_{2} \mathrm{OCH}$ ), 7.15-7.31 (m, 15H, phenyl H). ${ }^{13} \mathrm{C}$ NMR $\left(50 \mathrm{MHz}, \mathrm{CDCl}_{3}\right), \delta_{\mathrm{C}} 28.1,49.0,57.7,70.8,71.9,73.3$, 
73.7, 73.9, 81.3, 127.0, 127.5, 127.8, 128.1, 128.3, 128.6, 128.8, 137.5, 138.8, 157.3. Anal. Calcd. for $\mathrm{C}_{29} \mathrm{H}_{31} \mathrm{NO}_{4} \mathrm{~S}$ (489.62): C, 71.14; H, 6.38; N, 2.86, Found: C, 71.11; H, 6.36; N, 2.81. Rac-6-[(1R,2S,3S)-1,2,3,4-tetrakis(benzyloxy)butyl]-3a,4-dihydro-3H,6H-thieno[3,4c]isoxazole (4h). Colourless oil, yield 64\%. ${ }^{1} \mathrm{H}$ NMR $\left(200 \mathrm{MHz}, \mathrm{CDCl}_{3}\right), \delta_{\mathrm{H}} 3.48(\mathrm{~m}, 3 \mathrm{H}$, $\left.\mathrm{SCH}_{2} \mathrm{CH}\right), 3.74\left(\mathrm{~m}, 3 \mathrm{H}, \mathrm{BnOCH}_{2} \mathrm{CHCHCH}\right), 3.91(\mathrm{~m}, 1 \mathrm{H}, \mathrm{CH}), 4.29\left(\mathrm{~m}, 1 \mathrm{H}, \mathrm{NOCH}_{2}\right), 4.36(\mathrm{~m}$, $\left.1 \mathrm{H}, \mathrm{NOCH}_{2}\right), 4.41$ (dd, 1H, J=4.0, $\left.6.5 \mathrm{~Hz}, \mathrm{CHCHCH}\right), 4.68\left(\mathrm{~s}, 2 \mathrm{H}, \mathrm{CH}_{2} \mathrm{OCH}{ }_{2} \mathrm{Ph}\right), 4.72$ (d, 1H, $J=5.5 \mathrm{~Hz}, \mathrm{CHS}$ ), 4.89-4.99 (AB system, $2 \mathrm{H}, J=11.5 \mathrm{~Hz}, \mathrm{PhCH} \mathrm{H}_{2} \mathrm{OCH}$ ), 4.94-5.04 (AB system, $2 \mathrm{H}, J=11.5 \mathrm{~Hz}, \mathrm{PhCH}_{2} \mathrm{OCH}$ ), 5.07-5.17 (AB system, $2 \mathrm{H}, J=11.5 \mathrm{~Hz}, \mathrm{PhCH}_{2} \mathrm{OCH}$ ), 7.14-7.30 (m, 20H, phenyl H). ${ }^{13} \mathrm{C}$ NMR $\left(50 \mathrm{MHz}, \mathrm{CDCl}_{3}\right), \delta_{\mathrm{C}} 27.9,49.5,68.8,57.4,71.9,73.4,73.5$, 73.7, 77.2, 80.4, 80.5, 127.3, 127.4, 127.5, 127.9, 128.1, 128.3, 128.8, 129.1, 137.4, 137.8, 138.1, 138.2, 156.6. Anal. Calcd. for $\mathrm{C}_{37} \mathrm{H}_{39} \mathrm{NO}_{5} \mathrm{~S}$ (609.77): C, 72.88; H, 6.45; N, 2.30, Found: C, 72. $92 ; \mathrm{H}, 6.41 ; \mathrm{N}, 2.27$.

Rac-6-[(1R,2S,3R,4R)-1,2,3,4,5-pentakis(benzyloxy)pentyl]-3a,4-dihydro-3H,6H-thieno[3,4c]isoxazole (4i). Colourless oil, yield $60 \% .{ }^{1} \mathrm{H}$ NMR $\left(200 \mathrm{MHz}, \mathrm{CDCl}_{3}\right), \delta_{\mathrm{H}} 3.42-3.52(\mathrm{~m}, 3 \mathrm{H}$, $\left.\mathrm{SCH}_{2} \mathrm{CH}\right), 3.73(\mathrm{dd}, 1 \mathrm{H}, J=3.5,5.5 \mathrm{~Hz}, \mathrm{SCHCHO}), 3.77(\mathrm{~m}, 1 \mathrm{H}, \mathrm{CH}), 3.82\left(\mathrm{~m}, 2 \mathrm{H}, \mathrm{BnOCH}_{2}\right)$, $4.15\left(\mathrm{dd}, 1 \mathrm{H}, J=2.5,5.5 \mathrm{~Hz}, \mathrm{CH}_{2} \mathrm{CHCHCH}\right), 4.28-4.35\left(\mathrm{~m}, 2 \mathrm{H}, \mathrm{NOCH}_{2}\right), 4.41(\mathrm{~m}, 1 \mathrm{H}$, $C H \mathrm{OBn}$ ), 4.76 (d, $1 \mathrm{H}, J=5.5 \mathrm{~Hz}, C H \mathrm{~S}), 4.82-4.87$ (AB system, $2 \mathrm{H}, J=11.5 \mathrm{~Hz}, \mathrm{PhCH}_{2} \mathrm{OCH}_{2}$ ), 4.91-5.01 (AB system, $2 \mathrm{H}, J=11.5 \mathrm{~Hz}, \mathrm{PhCH} \mathrm{H}_{2} \mathrm{OCH}$ ), 4.95-5.05 (AB system, $2 \mathrm{H}, J=11.5 \mathrm{~Hz}$, $\mathrm{PhCH}_{2} \mathrm{OCH}$ ), 4.97-5.07 (AB system, $2 \mathrm{H}, J=11.5 \mathrm{~Hz}, \mathrm{PhCH} \mathrm{H}_{2} \mathrm{OCH}$ ), 5.06-5.16 (AB system, 2H, $\left.J=11.5 \mathrm{~Hz}, \mathrm{PhCH} \mathrm{H}_{2} \mathrm{OCH}\right), 7.11-7.32$ (m, 25H, phenyl H). ${ }^{13} \mathrm{C} \mathrm{NMR}\left(50 \mathrm{MHz}, \mathrm{CDCl}_{3}\right), \delta_{\mathrm{C}} 28.1$, 48.7, 56.4, 69.4, 71.8, 72.0, 72.9, 73.1, 73.3, 73.7, 79.8, 79.9, 80.6, 127.4, 127.7, 127.8, 127.9, 128.0, 128.4, 128.7, 129.0, 129.1, 137.4, 137.7, 137.8, 137.9, 138.1, 158.0. Anal. Calcd. for $\mathrm{C}_{45} \mathrm{H}_{47} \mathrm{NO}_{6} \mathrm{~S}$ (729.92): C, 74.05; H, 6.49; N, 1.92, Found: C, 74. 12; H, 6.41; N, 1.97.

General procedure for the oxidation of 3a,4,-tetrahydro-3H,6H-thieno[3,4-c]isoxazoles (4ai) with periodic acid in the presence of ferric chloride ${ }^{7}$ and then treatment with acetic anhydride under the Pummerer conditions

To a stirred solution of 3a,4,-tetrahydro-3H,6H-thieno[3,4-c]isoxazoles $4 \mathbf{a}-\mathbf{i}$ (1 mmol) and ferric chloride $(0.03 \mathrm{mmol})$ in $3 \mathrm{ml}$ of acetonitrile, periodic acid $(1.2 \mathrm{mmol})$ in $2 \mathrm{ml}$ of acetonitrile was added. After completion, the reaction mixture was evaporated to give a residue which was quenched by addition of a saturated aqueous solution of sodium thiosulfate and extracted with dichloromethane $(4 \times 3 \mathrm{ml})$. The dichloromethane extracts were washed with water, dried over magnesium sulfate, and evaporated to give a residue, which was treated with acetic anhydride (5 $\mathrm{ml}$ ) at $100^{\circ} \mathrm{C}$ for one $\mathrm{h}$. The reaction mixture was cooled at $\mathrm{r}$. $\mathrm{t}$. and then quenched by addition of ice. The separated layers was washed with saturated aqueous sodium bicarbonate followed by brine. The acetate layer was dried and evaporated in vacuum to give a residue which was chromatographed on column by using cyclohexane : ethyl acetate $=2: 1$ as eluant to give 1,3 dihydrothieno[3,4-c]isoxazoles 6a-i.

6-Methyl-1H,3H-thieno[3,4-c]isoxazole (6a). Colourless oil, yield 75\%. ${ }^{1} \mathrm{H}$ NMR (200 MHz, $\left.\mathrm{CDCl}_{3}\right), \delta_{\mathrm{H}} 1.92\left(\mathrm{~s}, 3 \mathrm{H}, \mathrm{CH}_{3}\right), 4.86\left(\mathrm{~s}, 2 \mathrm{H}, \mathrm{OCH}_{2}\right), 6.28(\mathrm{~s}, 1 \mathrm{H}, \mathrm{H} 4), 10.15(\mathrm{~s}, 1 \mathrm{H}, \mathrm{NH}) .{ }^{13} \mathrm{C}$ 
NMR $\left(50 \mathrm{MHz}, \mathrm{CDCl}_{3}\right), \delta_{\mathrm{C}} 12.6,67.9,114.5,123.1,125.5,149.1$. Anal. Calcd. for $\mathrm{C}_{6} \mathrm{H}_{7} \mathrm{NOS}$ (141.19): C, 51.04; H, 5.00; N, 9.92, Found: C, 51.11; H, 5.01; N, 9.98.

6-Ethyl-1H,3H-thieno[3,4-c] isoxazole (6b). Colourless oil, yield $72 \%$. ${ }^{1} \mathrm{H}$ NMR (200 MHz, $\left.\mathrm{CDCl}_{3}\right), \delta_{\mathrm{H}} 1.32\left(\mathrm{t}, 3 \mathrm{H}, J=7.5 \mathrm{~Hz}, \mathrm{CH}_{3}\right), 2.87$ (q, 2H, $\left.J=7.5,12.0 \mathrm{~Hz}, \mathrm{CH}_{2}\right), 4.98(\mathrm{~s}, 2 \mathrm{H}$, $\mathrm{H} 3 \mathrm{a}, \mathrm{b}), 6.30$ (s, 1H, H4), 10.20 (s, 1H, NH). $\left.{ }^{13} \mathrm{C} \mathrm{NMR} \mathrm{(50} \mathrm{MHz,} \mathrm{CDCl}_{3}\right), \delta_{\mathrm{C}} 15.9,21.3,69.7$, 112.7, 114.0, 123.9, 149.4. Anal. Calcd. for $\mathrm{C}_{7} \mathrm{H}_{9} \mathrm{NOS}$ (155.21): C, 54.17; H, 5.84; N, 9.02, Found: C, 54.21; H, 5.81; N, 9.08.

6-Phenyl-1H,3H-thieno[3,4-c]isoxazole (6c). Colourless oil, yield 75\%. ${ }^{1} \mathrm{H}$ NMR (200 MHz, $\left.\mathrm{CDCl}_{3}\right), \delta_{\mathrm{H}} 4.75(\mathrm{~s}, 2 \mathrm{H}, \mathrm{H} 3), 6.41(\mathrm{~s}, 1 \mathrm{H}, \mathrm{H} 4), 7.26-7.30(\mathrm{~m}, 5 \mathrm{H}$, phenyl H), $10.01(\mathrm{~s}, 1 \mathrm{H}, \mathrm{NH})$. ${ }^{13} \mathrm{C}$ NMR $\left(50 \mathrm{MHz}, \mathrm{CDCl}_{3}\right), \delta_{\mathrm{C}} 68.3,115.3,119.0,122.6,126.4,127.8,128.1,133.8,145.7$. Anal. Calcd. for $\mathrm{C}_{11} \mathrm{H}_{9} \mathrm{NOS}$ (203.26): C, 65.00; H, 4.46; N, 6.89, Found: C, 65.08; H, 4.41; N, 6.91 .

6-Benzyl-1H,3H-thieno[3,4-c]isoxazole (6d). Colourless oil, yield 70\%. ${ }^{1} \mathrm{H}$ NMR (200 MHz, $\left.\mathrm{CDCl}_{3}\right), \delta_{\mathrm{H}} 3.71\left(\mathrm{~s}, 2 \mathrm{H}, \mathrm{CH}_{2} \mathrm{Ph}\right), 4.79(\mathrm{~s}, 2 \mathrm{H}, \mathrm{H} 3 \mathrm{a}, \mathrm{b}), 6.17(\mathrm{~s}, 1 \mathrm{H},=\mathrm{CH}), 7.21-7.30(\mathrm{~m}, 5 \mathrm{H}$, phenyl H), 9.89 (s, $1 \mathrm{H}, \mathrm{NH}) .{ }^{13} \mathrm{C} \mathrm{NMR}\left(50 \mathrm{MHz}, \mathrm{CDCl}_{3}\right), \delta_{\mathrm{C}} 149.7,140.7,31.6,66.4,114.7$, 121.4, 125.8, 126.2, 128.7, 129.7. Anal. Calcd. for $\mathrm{C}_{12} \mathrm{H}_{11} \mathrm{NOS}$ (217.28): C, 66.33; H, 5.20; N, 6.05, Found: C, 66.28; H, 5.11; N, 6.08.

6-[(Benzyloxy)methyl]-1H,3H-thieno[3,4-c]isoxazole (6e). Colourless oil, yield 74\%. ${ }^{1} \mathrm{H}$ NMR $\left(200 \mathrm{MHz}, \mathrm{CDCl}_{3}\right), \delta_{\mathrm{H}} 4.13$ (s, 2H, $\left.\mathrm{OCH}_{2}\right), 4.33$ (s, 2H, $\left.\mathrm{CH}_{2} \mathrm{Ph}\right), 4.91$ (s, 2H, OCH -het), 6.31 (s, $1 \mathrm{H}, \mathrm{SCH}), 7.21-7.30\left(\mathrm{~m}, 5 \mathrm{H}\right.$, phenyl H), $9.90(\mathrm{~s}, 1 \mathrm{H}, \mathrm{NH}) .{ }^{13} \mathrm{C} \mathrm{NMR}\left(50 \mathrm{MHz}, \mathrm{CDCl}_{3}\right), \delta_{\mathrm{C}} 64.2$, $66.4,73.7,114.7,121.4,122.3,125.8,126.2,128.7,129.7,139.7,149.7$. Anal. Calcd. for $\mathrm{C}_{13} \mathrm{H}_{13} \mathrm{NO}_{2} \mathrm{~S}$ (247.31): C, 63.13; H, 5.30; N, 5.66, Found: C, 63.18; H, 5.31; N, 5.68.

6-[(1R)-1,2-bis(benzyloxy)ethyl]-1H,3H-thieno[3,4-c]isoxazole (6f). Colourless oil, yield 72\%. ${ }^{1} \mathrm{H}$ NMR $\left(200 \mathrm{MHz}, \mathrm{CDCl}_{3}\right.$ ), $\delta_{\mathrm{H}} 3.53$ (m, 2H, OCH $\mathrm{H}_{2} \mathrm{CHO}$ ), 4.36-4.53 (AB system, 2H, $J=11.5$ $\left.\mathrm{Hz}, \mathrm{PhCH}_{2} \mathrm{OCH}\right), 4.66\left(\mathrm{~s}, 2 \mathrm{H}, \mathrm{PhCH}_{2}\right), 4.86\left(\mathrm{~s}, 2 \mathrm{H}, \mathrm{OCH}_{2}\right), 4.98(\mathrm{~m}, 1 \mathrm{H},=\mathrm{CCHO}), 6.39(\mathrm{~s}, 1 \mathrm{H}$, $=\mathrm{CCH}), 7.25-7.38\left(\mathrm{~m}, 10 \mathrm{H}\right.$, phenyl H), $9.98(\mathrm{~s}, 1 \mathrm{H}, \mathrm{NH}) .{ }^{13} \mathrm{C} \mathrm{NMR}\left(50 \mathrm{MHz}, \mathrm{CDCl}_{3}\right), \delta_{\mathrm{C}} 66.6$, 70.4, 72.4, 74.2, 75.7, 114.5, 124.1, 125.3, 126.0, 125.5, 127.7, 128.4, 128.7, 136.9, 138.2, 149.1. Anal. Calcd. for $\mathrm{C}_{21} \mathrm{H}_{21} \mathrm{NO}_{3} \mathrm{~S}$ (367.46): C, 68.64; H, 5.76; N, 3.81, Found: C, 68.63; H, 5.78; N, 3.83 .

6-[(1R,2R)-1,2,3-Tris(benzyloxy)propyl]-1H,3H-thieno[3,4-c]isoxazole (6g). Colourless oil, yield 73\%. ${ }^{1} \mathrm{H}$ NMR (200 MHz, $\left.\mathrm{CDCl}_{3}\right), \delta_{\mathrm{H}} 3.51$ (m, 2H, OCH$\left.{ }_{2} \mathrm{CHO}\right), 4.09\left(\mathrm{~m}, 1 \mathrm{H}, \mathrm{CH}_{2} \mathrm{CHCH}\right)$, 4.52-4.56 (AB system, $\left.2 \mathrm{H}, J=11.5 \mathrm{~Hz}, \mathrm{PhCH}_{2} \mathrm{OCH}\right), 4.58$ (s, 2H, $\left.\mathrm{PhCH}_{2} \mathrm{OCH}_{2}\right), 4.60-4.70(\mathrm{AB}$ system, $\left.2 \mathrm{H}, J=11.5 \mathrm{~Hz}, \mathrm{PhCH}_{2} \mathrm{OCH}\right), 4.71\left(\mathrm{~s}, 2 \mathrm{H}, \mathrm{OCH}_{2}\right), 4.70(\mathrm{~m}, 1 \mathrm{H},=\mathrm{CCHO}), 6.27(\mathrm{~s}, 1 \mathrm{H}$, $=\mathrm{CCH}), 7.37-7.22\left(\mathrm{~m}, 15 \mathrm{H}\right.$, phenyl H), $9.65(\mathrm{~s}, 1 \mathrm{H}, \mathrm{NH}) .{ }^{13} \mathrm{C} \mathrm{NMR}\left(50 \mathrm{MHz}, \mathrm{CDCl}_{3}\right), \delta_{\mathrm{C}} 66.4$, $66.8,70.3,73.1,74.0,76.4,81.2,115.5,123.1,124.5,126.0,127.5,127.8,127.9,128.1,128.4$, 128.6, 128.7, 137.3, 137.6, 138.4, 148.8. Anal. Calcd. for $\mathrm{C}_{29} \mathrm{H}_{29} \mathrm{NO}_{4} \mathrm{~S}$ (487.60): C, 71.43; $\mathrm{H}$, 5.39; N, 2.87, Found: C, 71.48; H, 5.41; N, 2.89 .

6-[(1S,2R,3R)-1,2,3,4-Tetrakis(benzyloxy)butyl]-1H,3H-thieno[3,4-c]isoxazole

(6h).

Colourless oil, yield 70\%. ${ }^{1} \mathrm{H}$ NMR $\left(200 \mathrm{MHz}, \mathrm{CDCl}_{3}\right), \delta_{\mathrm{H}} 3.70\left(\mathrm{~m}, 2 \mathrm{H}, \mathrm{OCH}_{2} \mathrm{CHO}\right), 3.91(\mathrm{~m}$, $\left.1 \mathrm{H}, \mathrm{OCH}_{2} \mathrm{CH}\right), 3.98(\mathrm{~m}, 1 \mathrm{H}, \mathrm{CHCHCH}), 4.11(\mathrm{~m}, 1 \mathrm{H}, \mathrm{CHCHCH}), 4.42-4.58$ (AB system, 2H, $J$ 
$\left.=11.5 \mathrm{~Hz}, \mathrm{PhCH} \mathrm{H}_{2} \mathrm{OCH}\right), 4.49-4.51\left(\mathrm{AB}\right.$ system, $\left.2 \mathrm{H}, J=10.5 \mathrm{~Hz}, \mathrm{PhCH}_{2} \mathrm{OCH}_{2}\right), 4.59(\mathrm{~s}, 2 \mathrm{H}$, $\mathrm{PhCH}_{2} \mathrm{OCH}_{2}$ ), 4.61-4.69 (AB system, 2H, $J=11.5 \mathrm{~Hz}, \mathrm{PhCH} \mathrm{OCH}_{2} \mathrm{OC} .70$ (m, 1H, =CCHO), $4.85\left(\mathrm{~s}, 2 \mathrm{H}, \mathrm{OCH}_{2}\right), 6.40(\mathrm{~s}, 1 \mathrm{H},=\mathrm{CCH}), 7.20-7.37\left(\mathrm{~m}, 20 \mathrm{H}\right.$, phenyl H), $9.45(\mathrm{~s}, 1 \mathrm{H}, \mathrm{NH}) .{ }^{13} \mathrm{C}$ NMR $\left(50 \mathrm{MHz}, \mathrm{CDCl}_{3}\right), \delta_{\mathrm{C}} 66.4,69.8,70.4,73.2,73.5,73.6,75.5,78.5,82.4,113.8,124.0$, 124.2, 126.0, 127.4, 127.8, 127.9, 128.2, 128.3, 128.4, 128.6, 128.7, 137.4, 137.5, 137.7, 138.0, 149.3. Anal. Calcd. for $\mathrm{C}_{37} \mathrm{H}_{37} \mathrm{NO}_{5} \mathrm{~S}$ (607.76): C, 73.12; H, 6.14; N, 2.30, Found: C, 73.18; H, $6.13 ; \mathrm{N}, 2.28$.

6-[(1S,2R,3S,4S)-1,2,3,4,5-Pentakis(benzyloxy)pentyl]-1H,3H-thieno[3,4-c]isoxazole (6i). Colourless oil, yield 68\%. ${ }^{1} \mathrm{H}$ NMR $\left(200 \mathrm{MHz}, \mathrm{CDCl}_{3}\right), \delta_{\mathrm{H}} 3.67\left(\mathrm{~m}, 2 \mathrm{H}, \mathrm{OCH}_{2} \mathrm{CHO}\right), 3.83(\mathrm{~m}$, $\left.1 \mathrm{H}, \mathrm{OCH}_{2} \mathrm{CH}\right), 3.90(\mathrm{~m}, 1 \mathrm{H}, \mathrm{CHCHCH}), 4.03(\mathrm{~m}, 1 \mathrm{H}, \mathrm{CHCHCH}), 4.32(\mathrm{~m}, 1 \mathrm{H}, \mathrm{CHCHCH})$, 4.47 (s, 2H, PhCH $\mathrm{OCH}_{2}$ ), 4.39-4.46 (AB system, 2H, $J=10.5 \mathrm{~Hz}, \mathrm{PhCH}_{2} \mathrm{OCH}$ ), 4.53-4.48 (AB system, $2 \mathrm{H}, J=10.5 \mathrm{~Hz}, \mathrm{PhCH} \mathrm{H}_{2} \mathrm{OCH}$ ), 4.60-4.47 (AB system, $2 \mathrm{H}, J=11.0 \mathrm{~Hz}, \mathrm{PhCH}_{2} \mathrm{OCH}$ ), 4.51-4.71 (AB system, 2H, $\left.J=10.5 \mathrm{~Hz}, \mathrm{PhCH}_{2} \mathrm{OCH}\right), 4.87\left(\mathrm{~s}, 2 \mathrm{H}, \mathrm{OCH}_{2}\right), 5.02(\mathrm{~m}, 1 \mathrm{H}$, $=\mathrm{CCHO}), 5.91(\mathrm{~s}, 1 \mathrm{H},=\mathrm{CCH}), 7.20-7.36\left(\mathrm{~m}, 25 \mathrm{H}\right.$, phenyl H), $9.32(\mathrm{~s}, 1 \mathrm{H}, \mathrm{NH}) .{ }^{13} \mathrm{C}$ NMR $(50$ $\left.\mathrm{MHz}, \mathrm{CDCl}_{3}\right), \delta_{\mathrm{C}} 66.4,68.7,70.2,73.1,73.2,74.4,75.7,78.8,78.9,81.7,113.6,123.9,124.0$, 127.2 , 127.7, 127.8, 127.9, 128.1, 128.2, 128.3, 128.4, 128.6, 128.7, 136.9, 137.4, 137.9, 138.1, 138.8, 147.5. Anal. Calcd. for $\mathrm{C}_{45} \mathrm{H}_{45} \mathrm{NO}_{6} \mathrm{~S}$ (727.90): C, 74.25; H, 6.23; N, 1.92\%, Found: C, $74.28 ; \mathrm{H}, 6.21 ; \mathrm{N}, 1.95 \%$.

\section{General procedure for the reducing ring opening of isoxazolidine moiety of (6a-i) to give aminoalcohols (7a-i)}

To a solution of $1 H, 3 H$-thieno[3,4-c]isoxazoles 6a-i $(2 \mathrm{mmol})$ in $10 \mathrm{ml}$ of anhydrous THF, lithium aluminium hydride ( $4 \mathrm{mmol}$ ), suspended in $10 \mathrm{ml}$ of the same solvent (anhydrous THF), was added dropwise at room temperature. The mixture was refluxed for $2 \mathrm{~h}$ after the addition was complete, cooled and quenched by dropwise addition of $10 \mathrm{ml}$ of concentrated $\mathrm{Na}_{2} \mathrm{SO}_{4}$ solution. The mixture was then extracted with $\mathrm{CHCl}_{3}$ and organic extracts were combined and dried over $\mathrm{MgSO}_{4}$. After filtration, the removal of solvent under reduced pressure gave crude aminoalcohols which were initially crystallized from ethanol. However, the yields were low (50$56 \%$ ) due to their apparent instability, and therefore, they were not directly purified, but dissolved in absolute alcohol and the resulting solution added of gaseous hydrogen chloride. By addition of absolute ether a initial crop (25-28\%) of the corresponding chlorides precipitated and after standing one more day the filtrate deposited an additional $24-26 \%$ (total 49-54\%) of product.

(4-Amino-5-methyl-3-thienyl)methanol (7a). ${ }^{10}$ Colourless crystals, mp 72-74 ${ }^{\circ} \mathrm{C}$, yield 55\%, IR $\left(v_{\max } \mathrm{cm}^{-1}\right): 3360,2930,2850 .{ }^{1} \mathrm{H}$ NMR $\left(200 \mathrm{MHz}, \mathrm{CDCl}_{3}\right), \delta_{\mathrm{H}} 2.21\left(\mathrm{~s}, 3 \mathrm{H}, \mathrm{CH}_{3}\right), 4.25(\mathrm{bs}$, $1 \mathrm{H}, \mathrm{OH}), 4.37\left(\mathrm{~d}, 2 \mathrm{H}, J=6.5, \mathrm{CH}_{2} \mathrm{OH}\right), 6.38(\mathrm{~s}, 1 \mathrm{H}, \mathrm{H}-2) .{ }^{13} \mathrm{C} \mathrm{NMR}\left(50 \mathrm{MHz}, \mathrm{CDCl}_{3}\right), \delta_{\mathrm{C}}: 11.1$, 60.4, 115.4, 126.2, 126.3, 146.0. Anal. Calcd. for $\mathrm{C}_{6} \mathrm{H}_{9} \mathrm{NOS}(143,20)$ : C, 50.32, H, 6.33, N, 9.78\%, Found: C, 50.36; H, 6.31; N, 9. 18\%. 
(4-Amino-5-methyl-3-thienyl)methanol hydrochloride. Colourless crystals, mp 174-175 ${ }^{\circ} \mathrm{C}$. Anal. Calcd. for $\mathrm{C}_{6} \mathrm{H}_{9} \mathrm{NOS} \cdot \mathrm{HCl}$ (179.66): C, 40.11; H, 5.61; N, 7.80, Found: C, 40.14; H, 5.63; N, 7.84 .

(4-Amino-5-ethyl-3-thienyl)methanol (7b). Colourless crystals, mp 69-71 ${ }^{\circ} \mathrm{C}$, yield 58\%, IR $\left(v_{\max } \mathrm{cm}^{-1}\right): 3370,2930,2850 .{ }^{1} \mathrm{H}$ NMR $\left(200 \mathrm{MHz}, \mathrm{CDCl}_{3}\right), \delta_{\mathrm{H}} 1.27\left(\mathrm{t}, 3 \mathrm{H}, J=7.5 \mathrm{~Hz}, \mathrm{CH}_{3}\right)$, 2.64 (q, 2H, $\left.J=7.5,12.0 \mathrm{~Hz}, \mathrm{CH}_{2}\right), 4.29$ (s, 2H, $\left.\mathrm{CH}_{2} \mathrm{OH}\right), 5.31$ (bs, 2H, $\mathrm{NH}_{2}$ ), 6.29 (s, 1H, $=\mathrm{CH}) .{ }^{13} \mathrm{C}$ NMR $\left(50 \mathrm{MHz}, \mathrm{CDCl}_{3}\right), \delta_{\mathrm{C}} 16.36,20.49,60.17,113.66,117.21,124.59,146.33$. Anal. Calcd. for $\mathrm{C}_{7} \mathrm{H}_{11} \mathrm{NOS}$ (157.23): C, 53.47\%; H, 7.05\%; N, 8.91\%, Found: C, 53.51\%; H, $7.01 \% ; \mathrm{N}, 8.98 \%$.

(4-Amino-5-ethyl-3-thienyl)methanol hydrochloride. Colourless crystals mp $168-170^{\circ} \mathrm{C}$ Anal. Calcd. for $\mathrm{C}_{7} \mathrm{H}_{11} \mathrm{NOS} \cdot \mathrm{HCl}$ (193.69): C, 43.41; H, 6.24; N, 7.23\%, Found: C, 43.44; H, 6.22; N, $7.27 \%$.

(4-Amino-5-phenyl-3-thienyl)methanol (7c). Colourless crystals, $80-82^{\circ} \mathrm{C}$, yield $62 \%$, IR $\left(v_{\max }\right.$ $\left.\mathrm{cm}^{-1}\right): 3365,2920,2860 .{ }^{1} \mathrm{H}$ NMR $\left(200 \mathrm{MHz}, \mathrm{CDCl}_{3}\right), \delta_{\mathrm{H}} 4.35$ (d, 2H, $\left.J=13.0 \mathrm{~Hz}, \mathrm{CH}_{2} \mathrm{OH}\right)$, $5.31\left(\mathrm{bs}, 2 \mathrm{H}, \mathrm{NH}_{2}\right), 6.27(\mathrm{~s}, 1 \mathrm{H},=\mathrm{CH}), 7.31-7.28(\mathrm{~m}, 5 \mathrm{H}$, phenyl $\mathrm{H}) .{ }^{13} \mathrm{C} \mathrm{NMR}(50 \mathrm{MHz}$, $\left.\mathrm{CDCl}_{3}\right), \delta_{\mathrm{C}} 145.4,133.7,129.1,127.9,127.1,125.8,117.5,116.0,61.3,116.06,125.82,127.15$, 127.62, 128.37, 129.15, 133.68, 145.45. Anal. Calcd. for $\mathrm{C}_{11} \mathrm{H}_{10} \mathrm{NOS}$ (205.27): C, 64.36; H, $5.40 ; \mathrm{N}, 6.82$, Found: C,64.38; H, 5.42; N, 6.88 .

(4-Amino-5-phenyl-3-thienyl)methanol hydrochloride. Colourless crystals, $\mathrm{mp} 178-180{ }^{\circ} \mathrm{C}$. Anal. Calcd. for $\mathrm{C}_{11} \mathrm{H}_{10} \mathrm{NOS} \cdot \mathrm{HCl}$ (241.73): C, 54.65\%; H, 5.00\%; N, 5.79\%. Found: C, 54.70\%; H, 5.04\%; N, 5.73\%.

(4-Amino-5-benzyl-3-thienyl)methanol (7d). Colourless crystals, mp 76-77 ${ }^{\circ} \mathrm{C}$, yield $60 \%$, IR $\left(v_{\max } \mathrm{cm}^{-1}\right): 3380,2915,2870 .{ }^{1} \mathrm{H} \mathrm{NMR}\left(200 \mathrm{MHz}, \mathrm{CDCl}_{3}\right), \delta_{\mathrm{H}} 7.30-7.21(\mathrm{~m}, 5 \mathrm{H}$, phenyl H), $6.23(\mathrm{~s}, 1 \mathrm{H},=\mathrm{CH}), 5.31\left(\mathrm{bs}, 2 \mathrm{H}, \mathrm{NH}_{2}\right), 4.24\left(\mathrm{~s}, 2 \mathrm{H}, \mathrm{CH}_{2} \mathrm{OH}\right), 3.68\left(\mathrm{~s}, 2 \mathrm{H}, \mathrm{CH}_{2} \mathrm{Ph}\right) .{ }^{13} \mathrm{C} \mathrm{NMR}(50$ $\left.\mathrm{MHz}, \mathrm{CDCl}_{3}\right), \delta_{\mathrm{C}} 30.81,60.46,115.58,124.60,126.51,126.25,128.68,130.08,140.89,146.68$. Anal. Calcd. for $\mathrm{C}_{12} \mathrm{H}_{13} \mathrm{NOS}$ (219.30): C, 65.72\%; H, 5.97\%; N, 6.39\%, Found: C, 65.78\%; H, $5.92 \% ; \mathrm{N}, 6.32 \%$.

(4-Amino-5-benzyl-3-thienyl)methanol hydrochloride. Colourless crystals, mp $175-177^{\circ} \mathrm{C}$. Anal. Calcd. for $\mathrm{C}_{12} \mathrm{H}_{13} \mathrm{NOS} \cdot \mathrm{HCl}$ (255.76): C, 56.35\%; H, 5.52\%; N, 5.48\%. Found: C, 56.39\%; H, 5.54\%; N, 5.47\%.

\{4-Amino-5-[(benzyloxy)methyl]-3-thienyl\}methanol (7e). Amorfous solid, yield 60\%, IR $\left(v_{\max } \mathrm{cm}^{-1}\right) 3370,2930,2855 .{ }^{1} \mathrm{H} \mathrm{NMR}\left(200 \mathrm{MHz}, \mathrm{CDCl}_{3}\right), \delta_{\mathrm{H}} 4.50(\mathrm{~d}, 2 \mathrm{H}, J=13.0 \mathrm{~Hz}$, $\left.\mathrm{CH}_{2} \mathrm{OH}\right), 4.52\left(\mathrm{~m}, 2 \mathrm{H},=\mathrm{CCH}_{2}\right), 4.56$ (AB system, $\left.2 \mathrm{H}, J=12.0 \mathrm{~Hz}, \mathrm{CH}_{2} \mathrm{Ph}\right), 5.29$ (bs, $2 \mathrm{H}, \mathrm{NH}_{2}$ ), $6.36(\mathrm{~s}, 1 \mathrm{H},=\mathrm{CH}), 7.33-7.29\left(\mathrm{~m}, 5 \mathrm{H}\right.$, phenyl H). ${ }^{13} \mathrm{C} \mathrm{NMR}\left(50 \mathrm{MHz}, \mathrm{CDCl}_{3}\right), \delta_{\mathrm{C}} 145.6,137.8$, 128.4, 128.3, 127.5, 126.2, 125.2, 115.3, 73.3, 63.4, 60.2. Anal. Calcd. for $\mathrm{C}_{13} \mathrm{H}_{15} \mathrm{NO}_{2} \mathrm{~S}(249.32)$ : C, 62.62; H, 6.06; N, 5.62, Found: C, 62.68; H, 6.63; N, 5.68.

\{4-Amino-5-[(1R)-1,2-bis(benzyloxy)ethyl]-3-thienyl\}methanol (7f). Colourless oil, yield $58 \%$. ${ }^{1} \mathrm{H}$ NMR (200 MHz, $\left.\mathrm{CDCl}_{3}\right), \delta_{\mathrm{H}} 3.52$ (m, 2H, OCH $\mathrm{OCHO}_{2}, 4.32$ (s, 2H, $\left.\mathrm{CH}_{2} \mathrm{OH}\right), 4.34-$ 4.52 (AB system, $2 \mathrm{H}, J=11.5 \mathrm{~Hz}, \mathrm{PhCH} \mathrm{H}_{2} \mathrm{OCH}$ ), 4.65 (AB system, $2 \mathrm{H}, J=11.5 \mathrm{~Hz}$, $\left.\mathrm{PhCH} \mathrm{H}_{2} \mathrm{OCH}\right), 4.93(\mathrm{~m}, 1 \mathrm{H},=\mathrm{CCHO}), 5.31\left(\mathrm{bs}, 2 \mathrm{H}, \mathrm{NH}_{2}\right), 6.25(\mathrm{~s}, 1 \mathrm{H},=\mathrm{CCH}), 7.24-7.37(\mathrm{~m}$, 
$10 \mathrm{H}$, phenyl H). ${ }^{13} \mathrm{C}$ NMR $\left(50 \mathrm{MHz}, \mathrm{CDCl}_{3}\right), \delta_{\mathrm{C}} 146.1,138.3,136.9,128.9,128.3,127.6,127.2$, 126.9, 126.0, 125.9, 114.7, 75.7, 74.2, 72.4, 69.9, 60.2. Anal. Calcd. for $\mathrm{C}_{21} \mathrm{H}_{23} \mathrm{NO}_{3} \mathrm{~S}$ (369.47): C, 68.27; H, 6.27; N, 3.79, Found: C, 68.31; H, 6.23; N, 3.78.

\{4-Amino-5-[(1R,2R)-1,2,3-tris(benzyloxy)propyl]-3-thienyl\}methanol (7g). Colourless oil, yield $55 \%$. ${ }^{1} \mathrm{H}$ NMR $\left(200 \mathrm{MHz}, \mathrm{CDCl}_{3}\right), \delta_{\mathrm{H}} 3.52\left(\mathrm{~m}, 2 \mathrm{H}, \mathrm{OCH}_{2} \mathrm{CHO}\right), 4.05\left(\mathrm{~m}, 1 \mathrm{H}, \mathrm{CHCHCH}_{2}\right)$, 4.30 (s, $\left.2 \mathrm{H}, \mathrm{CH}_{2} \mathrm{OH}\right), 4.35-4.25$ (AB system, $\left.2 \mathrm{H}, J=11.5 \mathrm{~Hz}, \mathrm{PhCH}_{2} \mathrm{OCH}\right), 4.40-4.57$ (AB system, $2 \mathrm{H}, J=11.5 \mathrm{~Hz}, \mathrm{PhCH}_{2} \mathrm{OCH}$ ), 4.65-4.75 (AB system, $2 \mathrm{H}, J=11.5 \mathrm{~Hz}, \mathrm{PhCH}_{2} \mathrm{OCH}$ ), $4.91(\mathrm{~m}, 1 \mathrm{H},=\mathrm{CCHO}), 5.31\left(\mathrm{bs}, 2 \mathrm{H}, \mathrm{NH}_{2}\right), 6.24(\mathrm{~s}, 1 \mathrm{H},=\mathrm{CCH}), 7.24-7.37(\mathrm{~m}, 15 \mathrm{H}$, phenyl H). ${ }^{13} \mathrm{C}$ NMR $\left(50 \mathrm{MHz}, \mathrm{CDCl}_{3}\right), \delta_{\mathrm{C}} 145.8,138.5,137.7,137.4,128.7,128.6,128.4,127.8,127.7$, 126.5, 126.2, 125.7, 114.4, 81.5, 76.5, 74.1, 73.1, 69.8, 66.5, 60.1. Anal. Calcd. for $\mathrm{C}_{29} \mathrm{H}_{31} \mathrm{NO}_{4} \mathrm{~S}$ (489.62): C, 71.14; H, 6.38; N, 2.86, Found: C, 71.19; H, 6.34; N, 2.83.

\{4-Amino-5-[(1S,2R,3R)-1,2,3,4-tetrakis(benzyloxy)butyl]-3-thienyl\}methanol

(7h). Colourless oil, yield 54\%. ${ }^{1} \mathrm{H}$ NMR $\left(200 \mathrm{MHz}, \mathrm{CDCl}_{3}\right), \delta_{\mathrm{H}} 3.97(\mathrm{~m}, 1 \mathrm{H}, \mathrm{CHCHCH}), 4.11(\mathrm{~m}$, $1 \mathrm{H}, \mathrm{CHCHCH}), 4.28\left(\mathrm{~s}, 2 \mathrm{H}, \mathrm{CH}_{2} \mathrm{OH}\right), 4.39-4.51$ (AB system, $2 \mathrm{H}, J=11.5 \mathrm{~Hz}, \mathrm{PhCH}_{2} \mathrm{OCH}$ ), $4.54(\mathrm{~m}, 1 \mathrm{H},=\mathrm{CCHO}), 4.46-4.61\left(\mathrm{AB}\right.$ system, $\left.2 \mathrm{H}, J=11.5 \mathrm{~Hz}, \mathrm{PhCH} \mathrm{H}_{2} \mathrm{OCH}\right), 4.60-4.69(\mathrm{AB}$ system, $\left.2 \mathrm{H}, J=11.5 \mathrm{~Hz}, \mathrm{PhCH}_{2} \mathrm{OCH}\right), 4.51\left(\mathrm{~s}, 2 \mathrm{H}, \mathrm{PhCH}_{2} \mathrm{OCH}_{2}\right), 4.78\left(\mathrm{~s}, 2 \mathrm{H}, \mathrm{OCH}_{2}\right), 5.03(\mathrm{~m}$, $1 \mathrm{H},=\mathrm{CCHO}), 5.35\left(\mathrm{bs}, 2 \mathrm{H}, \mathrm{NH}_{2}\right), 6.25(\mathrm{~s}, 1 \mathrm{H},=\mathrm{CCH}), 7.23-7.37\left(\mathrm{~m}, 20 \mathrm{H}\right.$, phenyl H). ${ }^{13} \mathrm{C} \mathrm{NMR}$ $\left(50 \mathrm{MHz}, \mathrm{CDCl}_{3}\right), \delta_{\mathrm{C}} 145.3,138.80,137.8,137.5,137.1,128.7,128.6,128.3,128.2,128.1$, 127.9, 127.8, 127.6, 127.4, 125.9, 114.7, 82.7, 80.1, 77.7, 75.6, 74.7, 73.5, 72.7, 70.1, 69.7, 60.1. Anal. Calcd. for $\mathrm{C}_{37} \mathrm{H}_{39} \mathrm{NO}_{5} \mathrm{~S}$ (609.25): C, 72.88; H, 6.45; N, 2.30, Found: C, 72.81; H, 6.41; N, 2.27 .

\{4-amino-5-[(1S,2R,3S,4S)-1,2,3,4,5-pentakis(benzyloxy)pentyl]-3-thienyl\}methanol

(7i). Colourless oil, yield 54\%. ${ }^{1} \mathrm{H}$ NMR $\left(200 \mathrm{MHz}, \mathrm{CDCl}_{3}\right), \delta_{\mathrm{H}} 3.64\left(\mathrm{~m}, 2 \mathrm{H}, \mathrm{OCH}_{2} \mathrm{CHO}\right), 3.85(\mathrm{~m}$, $\left.1 \mathrm{H}, \mathrm{OCH}_{2} \mathrm{CH}\right), 3.98(\mathrm{~m}, 1 \mathrm{H}, \mathrm{CHCHCH}), 4.21(\mathrm{~m}, 1 \mathrm{H}, \mathrm{CHCHCH}), 4.30\left(\mathrm{~m}, 2 \mathrm{H}, \mathrm{CH}_{2} \mathrm{HO}\right), 4.32$ $(\mathrm{m}, 1 \mathrm{H}, \mathrm{CHCHCH}), 4.47\left(\mathrm{~s}, 2 \mathrm{H}, \mathrm{PhCH}_{2} \mathrm{OCH}_{2}\right), 4.38-4.56(\mathrm{AB}$ system, $2 \mathrm{H}, J=10.5 \mathrm{~Hz}$, $\mathrm{PhCH}_{2} \mathrm{OCH}_{2}$ ), 4.46-4.63 (AB system, $2 \mathrm{H}, J=10.5 \mathrm{~Hz}, \mathrm{PhCH}_{2} \mathrm{OCH}$ ), 4.53-4.66 (AB system, $2 \mathrm{H}$, $\left.J=11.0 \mathrm{~Hz}, \mathrm{PhCH} \mathrm{H}_{2} \mathrm{OCH}\right), 4.51-4.70\left(\mathrm{AB}\right.$ system, $\left.2 \mathrm{H}, J=10.5 \mathrm{~Hz}, \mathrm{PhCH} \mathrm{H}_{2} \mathrm{OCH}\right), 5.11(\mathrm{~m}, 1 \mathrm{H}$, $=\mathrm{CCHO}), 5.31\left(\mathrm{bs}, 2 \mathrm{H}, \mathrm{NH}_{2}\right), 6.15(\mathrm{~s}, 1 \mathrm{H},=\mathrm{CCH}), 7.14-7.35(\mathrm{~m}, 25 \mathrm{H}$, phenyl $\mathrm{H}) .{ }^{13} \mathrm{C}$ NMR $(50$ $\left.\mathrm{MHz}, \mathrm{CDCl}_{3}\right), \delta_{\mathrm{C}} 145.7,138.9,138.3,137.8,137.5,137.1,128.7,128.6,128.4,128.3,128.2$, 128.1, 127.9, 127.8, 127.7, 127.2, 126.0 124.0, 123.9, 114.7, 82.8, 80.8, 78.3, 74.6, 73.4, 72.7, 72.6, 70.3, 69.9, 60.0. Anal. Calcd. for $\mathrm{C}_{45} \mathrm{H}_{47} \mathrm{NO}_{6} \mathrm{~S}$ (729.31): C, 74.05; H, 6.49; N, 1.92, Found: C, 74.25; H, 6.51; N, 1.95 .

\section{Acknowledgements}

This research was partly supported by MIUR (Rome, Italy) in the frame of a PRIN project. Thanks are also due to CIMPS and CIRP consortia. 


\section{References}

1. For some recent examples of INOC processes, see: (a) Padwa, A.; Chiacchio, U.; Dean, D. C.; Schoffstall, A. M. J. Org. Chem. 1989, 54, 5277. (b) Chiacchio, U.; Corsaro, A.; Librando, V.; Rescifina, A.; Romeo, R.; Romeo, G. Tetrahedron 1996, 52, 14323. (c) Akritopoulou-Zanze, I.; Gracias, V.; Moore, J. D.; Djuric, S. W. Tetrahedron Lett. 2004, 45, 3421. (d) Park, K.-H.; Marshall, W. J. Tetrahedron Lett. 2004, 45, 4931. (d) Soro, Y.; Bamba, F.; Siaka, S.; Coustard, J.-M. Tetrahedron Lett. 2006, 47, 3315. (f) Shing, T. K. M.; Wong, Wai F.; Cheng, H. M.; Kwok, W. S.; So, K. H. Organic Lett. 2007, 9, 753. (g) Kadowaki, A.; Nagata, Y.; Uno, H.; Kamimura, A. Tetrahedron Lett. 2007, 48, 1823. (h) Kozikowski, A. P.; Park, P. U. J. Org. Chem. 1990, 55, 4668. (i) Ramachandiran, K.; Karthikeyan, K.; Muralidharan, D.; Perumal, P. T. Tetrahedron Lett. 2010, 51, 3006. (1) Shing, T. K. M.; So, K. H.; Kwok, W. S. Organic Lett. 2009, 11, 5070. (m) Bhosale, S.; Kurhade, S.; Prasad, Uppuleti V.; Palle, V. P.; Bhuniya, D. Tetrahedron Lett. 2009, 50, 3948. (n) Kamimura, A.; Yoshida, T.; Uno, H. Tetrahedron, 2008, 64, 11081.

2. See, for instance: (a) Morozov, D. A.; Kirilyuk, I. A.; Gatilov, Y. V.; Bagryanskaya, I. Y.; Bozhko, J. Y.; Komarov, D. A.; Grigor'ev, I. A.; Synthesis 2010, 2, 343. (b) Westermann, B.; Walter, A.; Florke, U.; Altenbach, H.-J. Organic Lett. 2001, 3, 1375. (c) Tranmer, G. K.; Tam, W. Organic Lett. 2002, 4, 4101. Gomez, A.; (d) Sandra. L.; Stashenko, E.; Palma, A.; Bahsas, A.; Amaro-Luis, J. M. Synlett 2006, 14, 2275

3. Corsaro, A.; Pistarà, V.; Chiacchio, M.A.; Vittorino, E.; Romeo, R. Tetrahedron Lett., 2007, $48,4915$.

4. (a) Hassner, A.; Dehaen, W. J. Org. Chem. 1990, 55, 5505. (b) Yan, M.-C.; Liu, J.-Y.; Lin, W.-W.; Kao, K.-H.; Liu,J.-T.; Jang, J.-J.; Yao, C.-F. Tetrahedron 1999, 55, 12493.

5. Mukaiyama, T.; Hoshino, T. J. Am. Chem. Soc. 1960, 82, 5339.

6. The mixture of diastereomer sulfoxides cannot be separated by chromatography.

7. Kim, S. S.; Nehru, S. K.; Kim, S.; Kim, D. W.; Jung H. C. Synthesis 2002, 2484.

8. Takahashi, M.; Daidouji, S.; Shiro, M.; Minakawa, N.; Matsuda, A. Tetrahedron 2008, 64, 4313.

9. To our best knowledge, compound $7 \mathbf{a}$ is reported as the sole product in the unique reference 10.

10. Wiedemann, F.; Kampe, W.; Dietmann, K.; Sponer, G. EP Patent 42593, 1981.

11. (a) Confalone, P. N.; Pizzolato, G.; Uskokovic, M. R.; Rouge, M. US Patent 4428963, 1984.

(b) Confalone, P. N.; Pizzolato, G.; Uskokovic, M. R.; Rouge, M. US Patent 4317915, 1982.

(c) Confalone, P.; Pizzolato, G.; Rouge, M.; Uskokovic, M. R. DE Patent 2737738, 1978.

(d) Barker, J. M.; Huddleston, P. R. EP Patent 298542, 1989, and references cited therein.

(e) Barker, J. M.; Huddleston, P. R. Eur. EP Patent 298542, 1989. (f) Barker, J. M.; Huddleston, P. R.; Wood, M. L. Synth. Comm. 2002, 32, 2565.

12. Geller, T. DE Patent 102004063191, 2006

13. Rippel, R.; Flemming, H. W.; Rukwied, M. DE Patent 3804794, 1989. 
14. Knochel, P.; Seebach, D. Synthesis 1982, 1017.

15. Varma, R. S.; Saini, R. K.; Meshram, H. M. Tetrahedron Lett. 1997, 38, 6525.

16. Kaczorowska, K.; Kolarska, Z.; Mitka, K.; Kowalski, P. Tetrahedron 2005, 61, 8315.

17. Hajipour, A. R.; Kooshki, B.; Ruoho, A E. Tetrahedron Lett. 2005, 46, 5503.

18. Shukla, V. G.; Salgaonkar, P. D.; Akamanchi K. G. J. Org. Chem. 2003, 68, 5422

19. (a) Yoshimoto, S.; Ichikawa, M.; Hildreth, J. E. K.; Ichikawa, Y. Tetrahedron Lett. 1996, 37, 2549; (b) Sears, P.; Wong, C. H. Chem. Commun. (Cambridge) 1998, 11, 1161.

20. Sowden, J. C.; Strobach, D. R. J. Am. Chem Soc. 1960, 82, 954.

21. Wolfrom, M. L.; Thompson, A. in Methods Carbohydr. Chem. 1963, 2, 427.

22. López-Herrera, F. J.; Sarabia-Garcia F. Tetrahedron 1997, 53, 3325.

23. Perrin, D. D.; Armarego, W. L. F.; Perrin, D. R. Purification of Laboratory Chemicals, 2nd Edn.; Pergamon: Oxford, 1980.

24. Parham, W. E.; Ramp, F. L. J. Am. Chem. Soc. 1951, 73, 1293. 